\title{
Using Advanced Simulation to Aid Microlithography Development
}

\author{
DANIEL C. COLE, EYTAN BAROUCH, EDWARD W. CONRAD, AND MICHAEL YEUNG
}

\author{
Invited Paper
}

\begin{abstract}
An early historical overview is first presented here on the use of simulation in optical microlithography, along with a description of the general physical models. This paper then turns to more recent development work in microlithography simulation, which has followed several very different tracts. Three of the most important areas are discussed here. The first involves improvements in the underlying physical models, such as advances beyond the Kirchhoff boundary condition in optical diffraction theory, as well as a deeper understanding into the chemistry and physical behavior of photoresist materials. Such work guides basic understanding both in the optics and photoresist areas. At the other extreme, phenomenological models are being advanced to enable simulation results on large scales to be placed in the hands of device and circuit designers. Finally, optimization of the large number of allowable parameters is a pervasive problem that has received much attention and interest by the engineering community.
\end{abstract}

Keywords-Lithography, microelectronics, microlithography, optics, simulation.

\section{INTRODUCTION}

Microlithography is the term often used in the semiconductor microchip industry when referring to the use of lithographic means to mass produce microelectronic chips. The basic technique roughly consists of exposing a thin film of a special type of material, called photoresist, to radiation or an electron beam, whereby the incident energy contains the spatial information necessary to pattern semiconductor device and circuit components. The photoresist has the special property that it will be chemically altered by the incident energy. A subsequent dissolution process then removes either the exposed or the unexposed film sections, depending on the

Manuscript received April 24, 2000; revised March 21, 2001. The work of D. C. Cole, E. Barouch, and M. Yeung was supported in part by the Defense Advanced Research Projects Agency and by the Air Force Office of Scientific Research.

D. C. Cole, E. Barouch, and M. Yeung are with the Department of Manufacturing Engineering, Boston University, Boston, MA 02215 USA (e-mail: dccole@bu.edu; eytan@bu.edu; myeung@bu.edu).

E. W. Conrad is with IBM Microelectronics, Essex Junction, VT 05452 USA (e-mail: econrad@us.ibm.com).

Publisher Item Identifier S 0018-9219(01)07246-2. type of photoresist material used. The final result is a complex pattern left in the film that closely matches the desired shapes of the semiconductor device and circuit element components that are to be fabricated. This patterned set of shapes in the film then serves as a mask for subsequent steps, such as ion implantation, etching, and deposition of other materials.

Microlithography is widely recognized as one of the most, if not the most, critical gating factors for enabling miniature semiconductor device structures to be manufactured on a truly massive scale. To emphasize this point, years before submicrometer structures could be manufactured in large quantities, technologists were able to create submicrometer semiconductor devices on a small scale basis, such as by patterning directly into photoresist with a scanning electron beam. However, mass producing such devices and making them available on a real commercial basis required far faster methods than "writing" the patterns of each device individually. Consequently, optical microlithography has been, and continues to be, the main workhorse for producing these small devices in large quantities, as this method involves the simultaneous illumination of massive number of device patterns. At some point in the future, microlithography techniques other than "optical" ones will need to be utilized. The question of when optical microlithography will no longer be adequate has been the subject of intense study and debate for many years now.

Certainly today the issue of small devices is no longer one of being "submicrometer." Present advanced manufacturable gate lengths in MOSFETs are about $0.18 \mu \mathrm{m}$, so the issue of "submicrometer" has long been settled. However, now the truly advanced devices that can be made on a single basis are approaching the nanometer regime. The same discrepancy exists now as in the submicrometer era: namely, despite the fact that individual devices with gate lengths smaller than $0.10 \mu \mathrm{m}$ can be created, they cannot presently be manufactured. Again, microlithography is the major gating factor.

The present paper will focus on a very specific aspect of optical microlithography, namely, the use of simulation to 
help guide the direction of this technology. Simulation has long been used to help turn novel lithographic techniques into manufacturable ones, as well as to fine-tune, optimize, and enable conventional and reasonably understood techniques to be used as aggressively as possible. We will first discuss all of these aspects here in some generality, and then we will turn in more detail to several areas that have been the focus of our own research for the past few years.

Engineers, technologists, and scientists from a variety of disciplines will find some commonality in the techniques used in simulating microlithography processes. (Reference [1], [2, Ch. 12-14], and [3, Ch. 7-9] serve as excellent introductions and overviews on microlithography.) For example, optics is, of course, a very important part of optical lithography, as is the more detailed understanding of the propagation of electromagnetic radiation through three-dimensional (3-D) microstructures. The solution to Maxwell's equations, and various approximations of this full solution, are important here, as also occurs in so many other areas of engineering, such as in the areas of microwaves, radar, and printed circuit boards. Virtually any communication application mediated by electromagnetic signals, such as occurs with the design of antennae and transmission lines, employs techniques similar to the ones to be discussed here for optical microlithography. Moreover, chemical engineers will find considerable overlap here with all the aspects of exposure and development of photoresist materials. Chemical reaction, diffusion, and surface evolution equations have all been prominent in describing the behavior of photoresists. Finally, microchip design engineers should find material here of considerable interest, since the deviations from the "straight-edge" designs that are used nearly exclusively in computer-aided design (CAD) circuit layouts, versus the actual fabricated semiconductor structures, can be understood and characterized in considerable detail by the methods described in the present paper.

Simulation methods are an important component of microlithography development for a number of reasons. First, the equipment involved in microlithography is extremely expensive, thereby necessitating careful planning when moving to the next generation of lithographic tools. For example, $\$ 5$ million to $\$ 10$ million for each exposure projection tool in a manufacturing line is not uncommon; likewise, having 20 such tools or more in a single leading-edge facility is not uncommon. These costs do not include the other critical costs of reticle making and photoresist processing. The entire toolset involved in lithographic processing is roughly limited in its ability to print smaller than a particular feature size. To produce printed dimensions smaller than this amount, when advancing to the next smaller device generation, requires either a major overhaul or a complete replacement of this tooling equipment. Such an investment by even the largest semiconductor manufacturers is daunting, and requires careful planning and checks to ensure that the investment will enable the desired goal to be reached. Simulation is one of the key tools used for making these checks, particularly prior to the availability of such equipment. Manufacturers of semiconductor chips need to predict future litho- graphic capabilities as accurately as possible, as this information influences all the other process changes (ion implantation, oxidation, diffusion, etching, deposition, etc.) that will jointly need to be made. In a similar vein, equipment manufacturers of the next-generation lithographic tools need to carefully guide and direct the design of these improved tools to fit the semiconductor manufacturer's future needs; simulation aids enormously here as well.

There is a second reason that simulation methods are important, even when a new semiconductor device generation is not being contemplated. When simple "shrinks" on present devices are being made, without major changes in the device design, then simulation is essential for determining the best way of pushing the present set of tooling to its maximum limits. For example, a number of optical enhancement "tricks" have been discovered during the past decade that can significantly improve the capability of available equipment. Part of this innovation has been born of necessity, since the development of a fully new generation of tooling at a new illumination wavelength or a completely new source of radiation, such as extreme ultraviolet (EUV) or X-ray, is an enormously expensive task.

Thus, simulation is helpful for long-term planning, when moving to an entirely new set of tooling, as well as for optimizing and pushing present microlithographic tooling to its most aggressive limits. However, we do not mean to imply that simulation is some sort of magical panacea. Continued experimentation in new areas is absolutely essential. The right balance between simulation and experiment is a difficult question to answer, and depends on many factors, such as the sophistication of the simulation program with respect to the phenomena being examined, the skill in using the program, the experimental factors that can be controlled, the skill and expertise of the experimenter, the availability of equipment, the relative expenses of the two approaches, etc. In general, both simulation and experimentation help each other test, confront, confirm, and probe each other's predictions and results. Often one or the other will produce results that are puzzling and in the end turn out to be mistakes in procedures; continued checks help to ensure that long periods of time are not spent on wrong directions. Some experimental results are absolutely critical for providing calibration or model parameters to a simulator. Some simulation results yield intermediate physical results that are nearly impossible to obtain experimentally, but that yield enormous physical insight into the inner workings of the mechanisms involved in the phenomena.

Having acknowledged this important point about experimentation, it is equally important to make the following point, namely, that "simulation" is our best means for making predictions about future possible experiments. Here, we mean "simulation" in a very global sense, and not "simulation" as pertains to some specific academic or commercial program that a technologist may or may not have found reliable. Simulation, in the broad sense, is an encoding of available information about some phenomena, and the structuring of it in such a way as to enable the best available replication, interpolation, or extrapolation of the 
observed phenomena under similar or different conditions. This "encoding of available information" means our best encapsulation of experimental measurements and theoretical knowledge, combined. If there is some new aspect of the phenomena that has been discovered, then what better way to record that knowledge than to fold it into the knowledge base and logic incorporated into a "simulation tool?" If a particular simulation tool is not "reliable," then probably either the phenomena is not well understood or characterized to begin with, perhaps over the regime of data in question, or else the software implementation of the available knowledge about the experiment has not been adequately carried out. Regarding the latter, we note that poor implementation can cover a wide range of categories, from very simple, basic coding mistakes, to user-friendly issues, to the very difficult problems of numerical robustness, stability, and convergence.

As will be emphasized here, simulation enters into microlithography at many different levels, ranging from the very fundamental level of physical description to the fairly phenomenological level. In some instances the more accurate physical description is desired, while in other situations less physically fundamental models may be desired. Specific examples will be provided here of these situations. Choosing the right level of physical detail depends on the intended objectives of the simulation, and the tradeoff between physical accuracy, robustness, computational time, memory requirements, and ease of use. Reference [4] discusses many of these points in some detail.

In this paper, we will first begin by providing a brief historical review on the development and use of simulation in microlithography, starting from the major beginning in the 1970s, and proceeding up to the most recent developments. Until only a few years ago, microlithography simulation was used nearly exclusively by lithography engineers; such software served as a guide for new engineering developments in perfecting the printability of microchip circuitry patterns. Most simulation aspects tended to concentrate on the imaging of light for a few lines of a mask, then simulating the effect of this imaging on the subsequent steps of exposing photoresist material, baking it, and dissolving the exposed or unexposed sections (depending on whether the resist was positive or negative, respectively). The use of simulation aided in deducing numerous "what-if" scenarios that were often considerably more difficult, time-consuming, and expensive to ascertain experimentally. While simulation in microlithography continues to be used in this way, there are also some new advances that promise to change the use of microlithography simulation, making it more a part of microchip design methods.

Section II-A first introduces the "conventional" use of microlithography simulation, and reviews much of its historical development. Section II-B then briefly describes more recent advances that promise to change the application of microlithography simulation. Sections III-V then turn to three key areas of active interest in microlithography that the authors have been directly involved. These areas are discussed specifically here both because of their importance to microlithography, and because they serve as illustrative examples of the range of simulation application, from the very physically detailed to the very phenomenological in nature. In particular, Section III will focus on a more fundamental physical description of imaging light through an inherently 3-D optical mask structure, by fully solving Maxwell's equations in the spatial and time domain as light propagates through the material. The importance of investigating the imaging process at this level of detail is discussed at some length. Section IV turns to a discussion of the effect of exposing, baking, and dissolving the exposed (unexposed) regions of the positive (negative) photoresist. Here, we describe recent work involving a very phenomenological model of the photoresist development process. Section $\mathrm{V}$ then addresses the importance of making use of modified illumination methods and optimization techniques to enhance the printability of projection optics. Recent results are discussed that hold promise for future applications. Section VI ends with some concluding remarks that tie these areas together.

\section{BRIEF HistoricAl ReVIEW}

\section{A. Early Work}

1) Overview: Lithographic techniques have long been used in many areas of mass replication of designs, both in art, as well as in engineering. Early on in the electronic industry, when printed circuit boards were such a prominent component, lithography was the means by which they were produced. After the transistor was invented in 1948, and later semiconductor integrated circuits (ICs) were developed, then extending the use of lithographic techniques to mass produce these circuits seemed a natural direction. However, many issues needed to be resolved that were not present in other areas of lithography.

Clearly the dimensions of device and circuit components in ICs were enormously smaller, namely, on the order of a few micrometers, than the corresponding dimensions for printed circuit boards, with dimensions on the order of millimeters. As can readily be imagined when making such a huge transition in dimensional size, early microlithographer investigators were not at all confident that imaging of optical mask patterns onto thin photoresist layers would be technically feasible. Issues on integrity of mask patterns, imaging near the optical limits, the ability of photoresists to adhere to semiconductor materials and to retain structural shape adequately to delineate such fine features, all had to be addressed. Moreover, the ICs necessitated other process steps not present with printed circuit boards, such as the need to mask out regions for ion implantation, for etching underlying semiconductor material, and for growing oxide in only specific regions to form insulation regions. Would photoresist materials enable such processes to be carried out? Indeed, they certainly have. Moreover, dimensions have shrunk enormously [5]-[7], falling from about $4 \mu \mathrm{m}$ in 1975 for the minimum critical dimensions, to about $0.18 \mu \mathrm{m}$ in 2000 .

Lithographers recognized early on that to push microlithography to the desired limits [8], then far more 


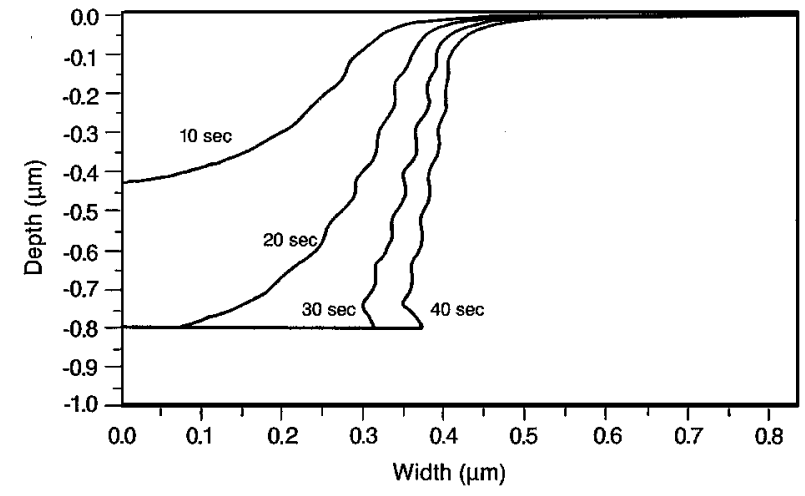

Fig. 1 Example of simulated photoresist contours created by SAMPLE, taken at 10-s intervals in the dissolution process.

needed to be understood about the detailed physical mechanisms in the different physical steps. An exciting period of early investigations led to a series of seminal papers [9]-[13] that were published by F. Dill's research group at IBM. These papers covered much of the basic physics and chemistry issues that enable a reasonable description of the key microlithographic processes, both from the standpoint of modeling and simulating the key phenomena, as well as measuring and characterizing the more critical physical parameters. Indeed, much of this work led to the development of the first major microlithography simulation program called SAMPLE, developed by A. Neureuther's research group at Berkeley [14], [15]. These developments in understanding of the interplay between imaging, exposing, baking, and dissolving, helped to take the "art" out of lithographic processes, and place the field in a much better technological and scientific position.

The early simulation programs, as best illustrated by the early academic program SAMPLE [14], [15] and later by some internal industrial programs and the commercial program PROLITH [16], [17], were all oriented toward understanding the formation of long lines and spaces in photoresist. More specifically, these programs calculated the two-dimensional (2-D) cross sections of infinitely long photoresist lines and spaces, as illustrated in Fig. 1.

Projection optics has been the dominant workhouse in microlithography, since the early beginnings of microelectronics. Each "level" of a microchip, such as the source and drain diffusion regions in a MOSFET, the high-conducting "gate" regions, the "contact hole" regions, the conducting lines connecting devices, etc., are all "printed" separately at each level, as imaged through optical masks that contain the entire pattern of shapes needed at that "level" in the microchip circuitry. The optical masks are reduced in size through the complex lens system that makes up the optical projection system.

A full calculation of the pattern transfer from the optical masks to the photoresist structure is enormously complicated and unrealistically computationally demanding for even the most powerful of today's computers. At the classical macroscopic level, such a calculation involves solving Maxwell's equations in time and space to ascertain the evolution of the full electromagnetic field due to the transmission of light through all the optical components in a microlithography optical projection system, and into the photoresist material. Several physical factors, however, enable many physical details to be ignored, thereby enormously simplifying this computation. First, the light used to illuminate the photoresist is quasi-monochromatic. Until the past decade or so in microlithography, this was accomplished by filtering the light from a powerful radiation source, such as a mercury arc lamp, to obtain the desired spectral component. Now, eximer laser sources are often used. Using quasi-monochromatic light means that the incident light is nearly all of the same frequency, so calculational means were able to be used that only dealt with essentially the change in time variation from the center monochromatic frequency. This change in time variation gives rise to the temporal coherency of the light, thereby providing a means to greatly simplify the calculations. Second, as will be discussed more in the following section, the average rate of absorption of the incident light by the photoresist occurs on a time scale that is approximately seven or more orders of magnitude slower than the periodicity of the incident light, as this rate is largely governed by the atomic and molecular bond transitions occurring in the resist due to the radiating light. Third, the macroscopic chemical and optical properties of the photoresist change much slower than even this time scale, thereby enabling the time evolution of the change in the photoresist material to be tracked at an even slower rate. The macroscopic absorption of energy by the photoresist material is governed by Poynting's theorem in classical electrodynamics [18], [19].

In addition to the time evolution approximations that were made in the early simulation work, two other approximations helped enormously to simplify calculations. The first was that the optical mask was very thin, so that it could be treated largely as a transmission function that simply acted to change the incident light amplitude by a multiplication function that varied as a function of space. As will be discussed more in Section III, many advanced masks being made today can no longer be treated in such a simple manner. Second, early optical projection systems had relatively low numerical apertures (NAs), at least as compared with today's systems. This fact enabled the rays of light incident on the photoresist to be treated as being nearly normally incident, thereby simplifying energy propagation into the photoresist.

In Sections II-A2-II-A5, we will discuss the early models used to describe the key microlithography steps. Although significant work has certainly been done since this very early work, these initial models form the basis for much of the work that has followed in subsequent years. By carefully reviewing this work, a better appreciation for subsequent work can be obtained. Moreover, as will be noted, considerable care is needed to be made in extracting the right level of physical detail at each of the process steps to adequately simulate the overall process. Section II-B will present a brief overview on the progress that has been made in academia and industry since this early work, in the areas of physical description, numerical computational ability, and ease of use. Sections III-V will go into much more detail regarding three specific areas of recent development. 
2) Imaging: The low NA of early projection systems enabled early researchers to separate the exposure of the resist into two parts, where first the average radiation energy was calculated as a function of space on the top surface of the photoresist, and then that information was used to approximate the propagation of the light energy into the resist. Moreover, the low NA assumption meant that the incident rays of light were at a sufficiently low angle of incidence to the normal to the photoresist that the coupling of the different vectorial components of the electromagnetic field could largely be ignored. Instead, a "scalar" optical imaging calculation was utilized, as discussed in [20]-[22].

For such a projection system, arguably the most important component for simulating the final shape of the photoresist structures is the calculation of the incident light energy distribution onto the photoresist surface. Often this quantity is referred to in microlithography as the "aerial image," in reference to the fact that the intensity of the light is in a plane at the top of the photoresist, prior to entering into the resist.

Fortunately, the mathematics for dealing with calculating an aerial image of an optical mask in a projection optics system was developed by $\mathrm{H}$. Hopkins nearly 50 years ago [23]. The key relevant parameters are: 1) the distance from the focal plane of the Gaussian image plane, meaning the distance to the plane where the best plane of focus exists, as determined by geometrical ray optics; 2) the center wavelength of the quasi-monochromatic light source; 3 ) a measure of degree of spatial partial coherence of the illumination system; 4) the NA of the side of the lens system illuminating the semiconductor wafer; 5) the aberrations of the optical system; and 6) a full description of the spatial transmission function representing the optical mask.

Early simulation work, such as implemented in SAMPLE [14], [15] and PROLITH [16], [17], were largely concerned with fairly simple optical masks consisting of only a few essentially infinitely long lines and spaces, or a periodic set of such lines and spaces. As will be discussed more in Sections III-V, the calculations of the aerial image of large 2-D mask pattern designs are now routinely done. However, such calculations have required the development of highly sophisticated algorithms to minimize the computational burdens [24], [25].

3) Exposure: The exposure process of a photoresist material refers to the act of light changing a photoresist material chemically, by breaking molecular bonds. The chemical change in the photoresist material alters the optical properties of the material as well, most notably by changing its absorption property. The model for the absorption coefficient that was proposed in 1975 [9], [10], [12] was the following simple phenomenological linear model:

$$
\alpha=A M(\mathbf{z}, \mathbf{t})+B
$$

where $M(\mathbf{z}, \mathbf{t})$ is the fraction of photosensitive material, per unit volume, present at position $\mathbf{z}$ and time $t$. The parameter $B$ equals the absorption coefficient when all photosensitive material has been chemically altered, while $A$ is the coefficient that dictates the linear dependence of $\alpha$ on $M(\mathbf{z}, \mathbf{t})$.
Both $A$ and $B$ are measured and characterized according to the type of photoresist being considered [10].

Equation (1) relates the concentration $M(\mathbf{z}, \mathbf{t})$ of the photoactive compound (PAC) to the absorption coefficient. However, a second important component is also necessary in order to model the exposure, namely, the time rate of change of $M(\mathbf{z}, \mathbf{t})$ as the photoresist material is exposed. The model used in 1975 was [9], [10], [12]

$$
\frac{\partial M}{\partial t}=-I(\mathbf{z}, \mathbf{t}) M(\mathbf{z}, \mathbf{t}) C
$$

so that the time-rate of change of $M(\mathbf{z}, \mathbf{t})$ is assumed to be linearly proportional to both the electromagnetic energy density $I(\mathbf{z}, \mathbf{t})$ at position $\mathbf{z}$ and time $t$ in the photoresist, as well as the amount of PAC present.

Thus, as the resist is exposed, a chemical reaction takes place in the photoresist, and the optical properties of the material change with time. The propagation of light into the resist needed to be taken into account. Standing waves form in the resist, due to an effective infinite number of reflections from the substrate/resist interface and the top surface of the photoresist [26]. The above model for positive photoresists, roughly described here, became known as Dill's $A$, $B, C$ model. [10] described how independent measurements could be made to determine these coefficients for each type of photoresist material.

4) Baking: Due to the fact that nearly monochromatic light is used to expose photoresist, standing wave light intensity patterns exist in the photoresist during exposure. If the photoresist is developed after such an exposure, then the walls of the photoresist structures will have many ripples in them, as illustrated in Fig. 1. As was discussed in [13], by performing a postexposure "baking" of the photoresist, the regions of high concentrations of photoactive compound concentration (PAC) can be "diffused" into the regions of low concentration, thereby smoothing out the PAC profile and removing the ripples. By doing this, a better process window is obtained, since then changes in the thickness of the resist, for example, will not result in such drastic changes in lateral width of developed lines due to ripple changes affecting profiles.

The early simulation methods used are still largely the same ones used today, namely, simply solving a diffusion equation that roughly described this baking process. However, certainly numerical methods have improved considerably [27] since early prototype approaches, and examinations have begun for nonlinear diffusion effects.

5) Dissolution: The early means for simulating the dissolution of the exposed part of the positive resist was by treating the development as though it occurred as a surface limited etching reaction, the rate of which depended on the value of $M$ at the surface. As described in [9], [10], and [12], by independently measuring the rate of dissolution $R(M)$ as a function of $M$, where an entire bulk film was prepared and exposed to have the same PAC concentration throughout, then a local rate $R[M(\mathbf{z}, \mathbf{t})]$ could be inferred and treated as the actual dissolution rate at each point on the surface of a film with varying PAC concentration. The work in [12] describes 
the mathematical treatment of the surface evolution as determined by dividing the film into small cells, and removing cells at a rate proportional to $R[M(\mathbf{z}, \mathbf{t})]$.

SAMPLE later made use of a string [28] and ray [29] algorithmic approaches to speed up and reduce the memory requirements of the cell method, although these approaches still continued to have difficulties. For simple cross sections, Fig. 1 illustrates what one would expect to obtain. From a numerical point of view, surface evolution is undoubtedly the most difficult of the computational aspects of photoresist processing simulation. Early difficulties arose because evolving surface fronts formed additional "loops." If the topology of the surface changed, even greater problems arose. Fortunately, considerable advances involving the mathematical and numerical treatment of surface evolution has occurred since the start of this work, as discussed in [27], [30]-[40], and in the level-set work in [41] and [42].

\section{B. More Recent Developments in Microlithography Simulation}

Since the mid-1980s, a number of advances have been made, particularly: 1) in the area of new physical conditions, such as for high NA situations [43]-[46], [25], which involves the inherent vector propagation of electromagnetic radiation [47], [48]; 2) the very significant advances in numerical methods, such as enabling 3-D solutions over nonplanar topographies [33], [35] for tackling "reflective notching" problems; and 3) the characterizations of new photoresist chemistries [49], [50], [1] and optical conditions [51], [52]. Regarding 3), new photoresist materials and processes were developed, such as negative, silylated, and chemically amplified photoresists, ${ }^{1}$ all requiring new simulation models and capabilities. Also, new means of modifying optical conditions [52], [53] also resulted in the need for improved simulation tools.

There are two nearly opposite directions that microlithography simulation is taking, as described in Sections III and IV. First, as situations become more complex, a more detailed account needs to be taken of physical situations and conditions. Such is the case as described in Section III, where advances are discussed that go beyond the usual calculation of the imaging of light through thin masks in optical projection systems. The introduction of "phase-shift" masks has been clearly shown, both experimentally and theoretically, to offer significantly enhanced resolution printing capability. However, such masks introduce material and topography variations that often violate the "thin mask" transmission approximation normally assumed in aerial image calculations. Section III describes advances by other researchers and ourselves on overcoming this obstacle.

Second, at the other extreme, developers often seek computationally simpler models that do not capture all of the physical details that a full computation is capable of including, simply due to the lack of time and memory resources. Section IV describes research on photoresist

\footnotetext{
${ }^{1}$ See, e.g., C. Grant Willson, "Organic Resist Materials," in [1], or further information mentioned in [49] and [50].
}

development and etch development models that has successfully followed this path. This approach may help bridge the fairly large gap that presently exists between microlithographers and microchip designers. We provide a number of comparisons between simulation and experiment here.

A third very important area of simulation that is attracting increasing attention is discussed in Section $\mathrm{V}$, namely, where automatic and semiautomatic means are pursued to enable large-scale optimization of the printability of microlithography processes. Such optimization procedures have been used with both the more physically fundamental microlithography simulation approaches, as well as the more phenomenological approaches. Section V discusses some of our own results to illustrate how dramatically subtle optical microlithography enhancements can be used to improve printability. This area emphasizes the optimization of a multitude of complex, but promising competing resolution enhancement effects. Each of these novel techniques can affect the other one in an often very nonlinear manner, so careful attention to the combination of these effects must be made in order to achieve maximum printability effects.

\section{Full SOLUTION OF MAXWELL's EQUATIONS FOR IMAGING Light Through A COMPLEX MASK STRUCTURE}

The microlithography process involves various scattering phenomena, such as the scattering of light from alignment targets during the pre-exposure alignment of wafers and the scattering of light through mask apertures during photoresist exposure. A full solution of Maxwell's equations first found its way into microlithography in connection with the problem of optical linewidth measurement of printed photoresist lines, where such a full solution was necessary to obtain an accurate description of the light scattering phenomenon involved [54]. The numerical technique employed was the waveguide method, so called because the fields within the object, which is assumed to be singly periodic (periodic in one direction) and to have a rectangular sidewall profile, are expanded in the eigenmodes of a waveguide having the same horizontal cross section as the object. The waveguide method was later extended to objects with arbitrary sidewall profiles [55] and applied to the modeling of alignment-target scattering, linewidth measurement and scattering from wafer topography [56]. Tanabe [57] extended the waveguide method to 3-D by formulating the problem in terms of the electromagnetic vector potential instead of the electric field. This approach was implemented in a complete 3-D photolithography simulator for modeling light scattering through mask apertures and from wafer topography during photoresist exposure [58].

A second approach based on the finite element method (FEM) was employed by Matsuzawa to solve the 2-D Helmholtz equation describing the scattering of light from wafer topography [59]. They showed that this kind of scattering can give rise to reflective notching, where the sidewall profile of a printed photoresist line in the vicinity of the topography is distorted by unwanted exposure caused by the scattered light. Urbach and Bernard extended the 
FEM approach to include the effects of partial coherence in the incident illumination and applied it to 2-D photoresist exposure simulation [60]. Barouch employed the spectral element method to model light scattering through mask apertures and from wafer topography [33].

A third approach based on the differential method [61] was employed by Yeung to model the scattering of light by inhomogeneity in a planar photoresist layer caused by beaching, or the change of the attenuation index, of the photoresist during exposure [43]. This method was extended to a photoresist layer with a singly periodic, nonplanar profile using a coordinate transformation technique [62]. It was applied to the simulation of light scattering from 2-D wafer topography [63].

A fourth approach based on the finite difference time domain (FDTD) method was employed by Wojcik to compute the scattering of light by small particles on a wafer surface [64]. This method was applied to the problems of alignmenttarget scattering [65] and linewidth measurement [66]. Guerrieri implemented a 2-D FDTD algorithm on the Connection Machine and applied it to simulate light scattering from 2-D wafer topography [67]. Wong extended the implementation to 3-D and applied it to the problem of light scattering through mask apertures [68], [69].

Lastly, the integral equation method was used by Kleemann to simulate light scattering from 2-D wafer topography [70]. Yeung used a periodic fast multipole method to model scattering from 3-D wafer topography [71].

Of the above-mentioned light scattering problems in microlithography, only the scattering of light by alignment targets, linewidth measurement and light scattering through mask apertures continue to receive attention today in the lithography community. The problem of light scattering by wafer topography has become of less importance due to the successful and widespread use of antireflective layers to suppress light scattering during exposure [72]. The following discussion will focus on the problem of light scattering through mask apertures, although the numerical techniques used to solve this problem are equally applicable to the problems of alignment-target scattering and linewidth measurement.

The computation of an aerial image requires knowledge of the field distribution on the mask plane due to the light transmitted through the mask apertures. In the usual treatment of aerial image formation, the field distribution on the mask plane is assumed to be that given by the Kirchhoff boundary conditions, ${ }^{2}$ in which scattering effects are neglected. Fig. 2(a) illustrates a Kirchhoff field distribution in the vicinity of a mask aperture whose dimensions are $2 \lambda \times$ $12 \lambda$, where $\lambda=157 \mathrm{~nm}$ is the wavelength of the incident light. In this figure, each point in the incident plane wave is assumed to propagate according to the laws of geometrical optics through the mask structure, so that diffraction through the mask aperture is absent. On the other hand, the field distribution computed by the FDTD method is shown Fig. 2(b), for a normally incident plane wave polarized in the

\footnotetext{
${ }^{2}$ See [20 p. 379].
}

(a) Kirchhoft field

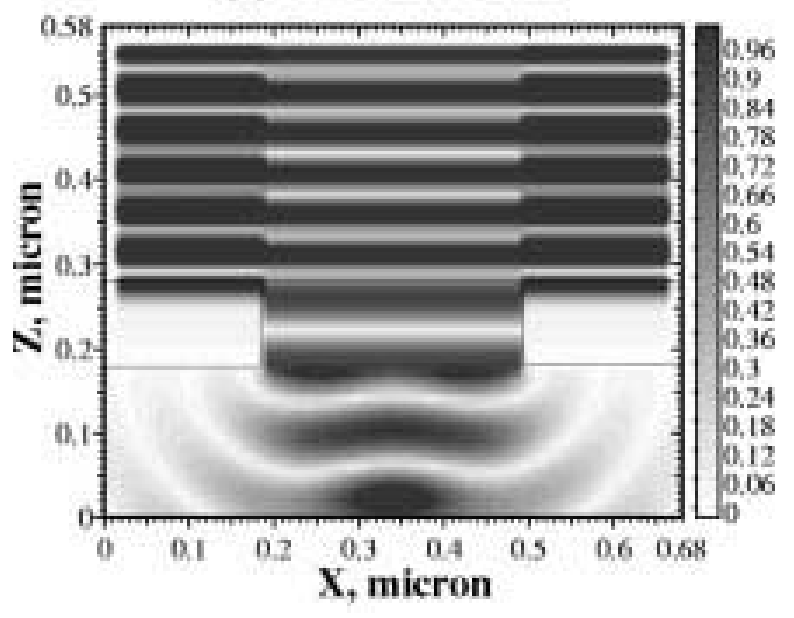

(b) FDTD field

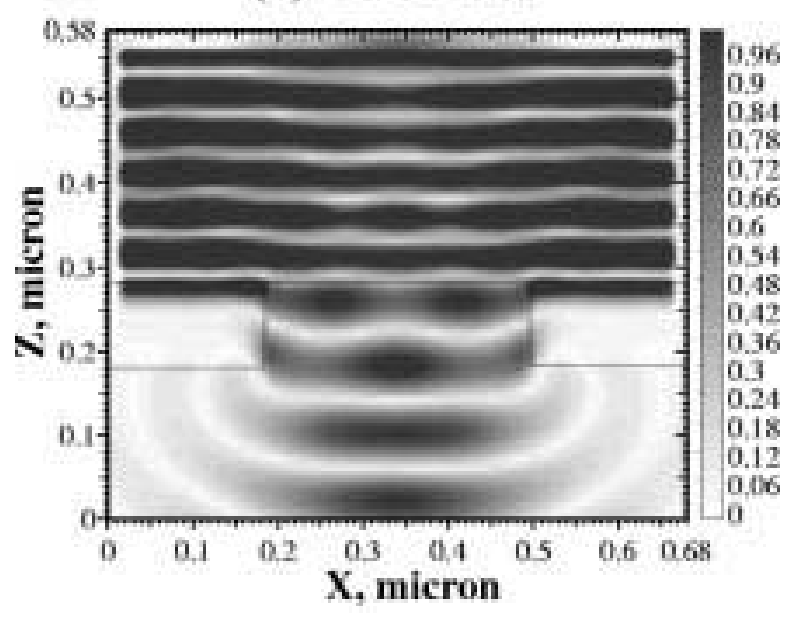

Fig. 2 Magnitude of the instantaneous electric field in the vicinity of a $2 \lambda$ by $12 \lambda$ isolated aperture in a binary mask illuminated by a normally incident plane wave polarized in the $X$ direction (TM). $\lambda=157 \mathrm{~nm}$, chromium thickness $=100 \mathrm{~nm}$, chromium refractive index $=0.68-1.11 j$. (a) Result based on the Kirchhoff boundary conditions. (b) Result computed by FDTD.

$y$ direction. It can be seen from these figures that there are significant differences between the Kirchhoff field distribution and the more accurate FDTD field distribution. When the Kirchhoff and FDTD field distributions are used for aerial image computation, the results can be very different, as illustrated in Fig. 3 for the mask structure of Fig. 2 and for a partially coherent optical system with $N A=0.7$ and partial coherence factor $\sigma=0.8$. Discrepancies between Kirchhoff and FDTD aerial images have been reported before in aerial image simulation in 248-nm lithography [73], [74]. The discrepancies shown in Figs. 2 and 3 for 157-nm lithography, however, are much larger. This is because the attenuation index of the chromium absorbing layer at $157 \mathrm{~nm}$ is smaller than that at $248 \mathrm{~nm}$ by a factor $1.11 / 2.01$ [75]. Therefore, to achieve the same attenuation factor, the ratio of the chromium thickness to the wavelength in a $157-\mathrm{nm}$ mask should be $2.01 / 1.11=1.8$ times that in a $248-\mathrm{nm}$ mask. This increased ratio leads to greater diffraction effects in the propagation of light through a mask aperture in 157-nm lithography and thus 
(a) TE Aerial Image

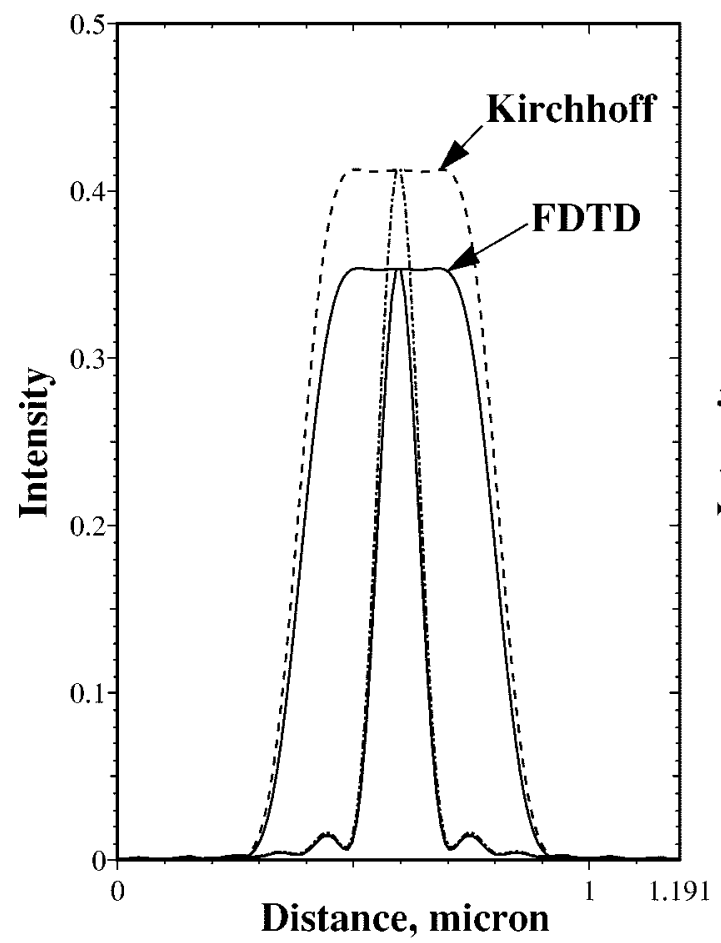

(b) TM Aerial Image

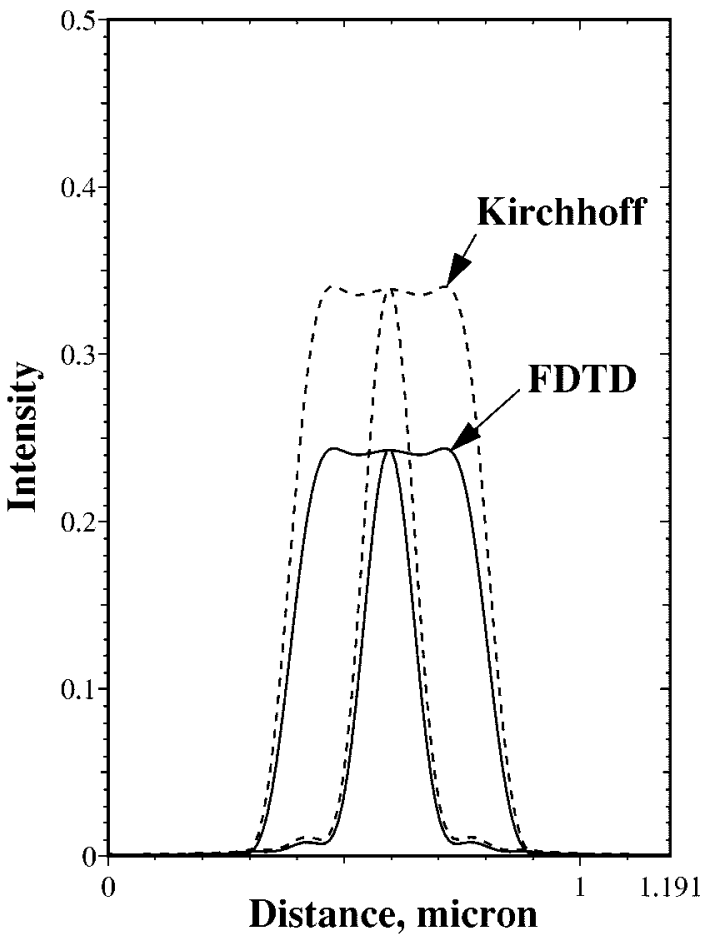

Fig. 3 Aerial images of a $0.5 \lambda$ by $3 \lambda$ isolated feature computed by FDTD and by the Kirchhoff boundary conditions. The cross sections of the aerial images along the width and length of the feature are shown. $\lambda=157 \mathrm{~nm}, N A=0.7, \sigma=0.8$, reduction ratio $=4: 1$, chromium thickness $=100 \mathrm{~nm}$. (a) TE polarization. (b) TM polarization.

greater discrepancy between the Kirchhoff and FDTD aerial images.

As can be seen from Fig. 3, the main difference between the Kirchhoff and FDTD aerial images, at least to first order, can be described by simply a scale factor. However, the value of this scale factor will vary from one situation to another, depending, for example, on the size of the aperture opening relative to the wavelength. Indeed, for complicated structures, the value of the approximate scale factor can depend on a large number of geometrical and physical factors concerning the mask (e.g., thickness and material composition) and the illuminating system itself (e.g., partial coherence, NA, and wavelength). For any given structure, the reason why an approximate scale factor difference exists between the Kirchhoff and FDTD aerial images is that the aerial images formed by a high NA optical system, such as those shown in Fig. 3, are determined primarily by the lowest few diffraction orders, which can be characterized by an effective transmission and an effective phase for each mask aperture involved [74]. However, the effective transmission and phase are dependent on the dimensions and thickness of the mask aperture compared to the wavelength, as well as the polarization of the incident light [76]. For general structures and situations, the approximate scale factor between the Kirchhoff and FDTD aerial images will have to be determined by simulation separately for each distinct mask geometry.

The above example illustrates how scattering from mask topography can have a major impact on the performance of binary as well as phase-shifting masks in 157-nm lithog- raphy. A full solution of Maxwell's equations for the propagation of light through the mask apertures is required to give the correct aperture fields for aerial-image simulation. As mentioned above, various numerical techniques have been used for the solution of this problem, the most popular of which are the waveguide method [58] and FDTD [69]. In the waveguide method, an arbitrarily shaped object is modeled by dividing it into a number of horizontal slabs and approximating each slab by a portion of a vertical waveguide having constant cross-sectional shape. In the FDTD method, an arbitrarily shaped object is modeled by a staircase shaped object conforming to the brick-like FDTD mesh. As a result, neither of these methods is suitable for the accurate modeling of objects with curved surfaces, such as the apertures of phase-shifting masks fabricated by a combination of isotropic and anisotropic etching [69].

Recently, a hybrid time-domain method combining the flexibility of the finite-element time domain (FETD) method in modeling curved surfaces with the computational efficiency of FDTD was developed by Wu and Itoh [77]. In this hybrid FETD-FDTD method, the region next to the curved surface of an object is treated by FEM while the remaining, smooth region is treated by FDTD. The two regions overlap in a thin transition region in which the fields are interpolated back and forth between the FEM and FDTD meshes during the time-marching loop of the algorithm. The original hybrid FETD-FDTD method of [77] is only applicable to lossless dielectric objects. The materials encountered in deep ultraviolet lithography, however, are often lossy, as in the case of 


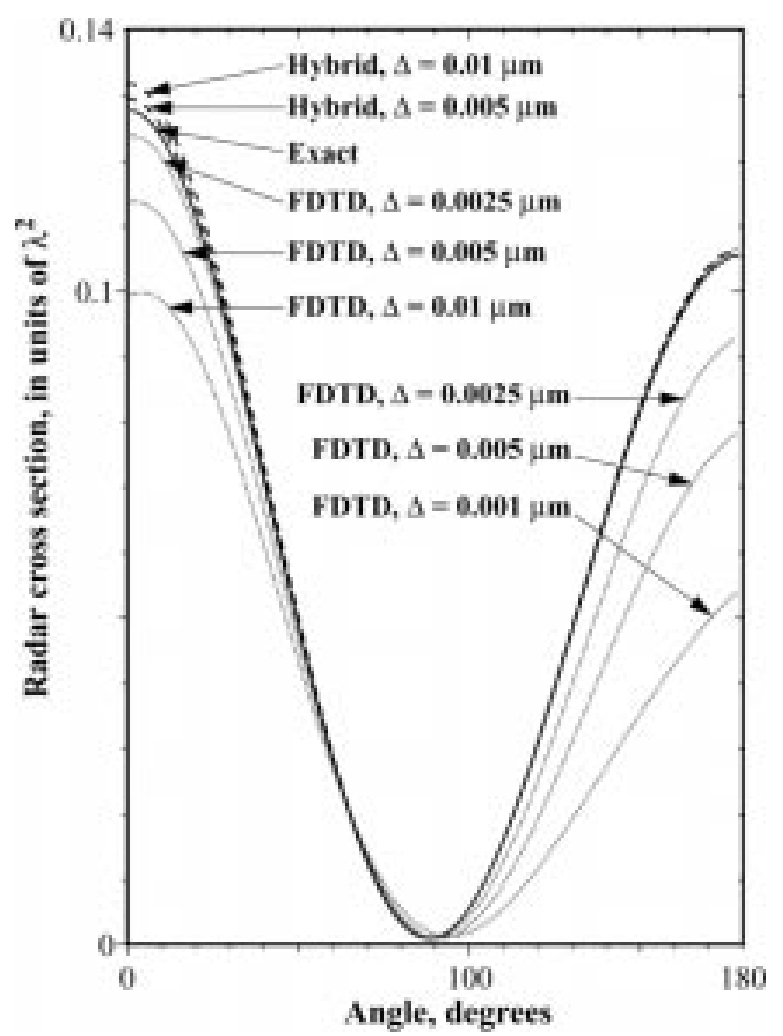

Fig. 4 Radar cross section of a sphere of radius $0.03 \mu \mathrm{m}$ and refractive index $0.85-2.01 j$ at a wavelength of $0.248 \mu \mathrm{m}$. The results computed by the hybrid FETD-FDTD and the FDTD methods for different mesh sizes $\Delta$ are compared with the exact result.

photoresist, and, furthermore, can have negative dielectric constants, as in the case of chromium. To handle such lossy materials, the original hybrid FETD-FDTD method has been extended to allow the use of a plasma permittivity function to model materials with negative dielectric constants [78].

The accuracy of the hybrid FETD-FDTD method has been verified by comparison with the exact result for a homogeneous spherical object. Fig. 4 shows the computed radar cross section, which is proportional to the amount of energy scattered per unit solid angle, as a function of the angle of scattering, for a sphere of refractive index $0.85-2.01 j$ and radius $0.03 \mu \mathrm{m}$, which is modeled by a plasma permittivity function, at a wavelength of $0.248 \mu \mathrm{m}$. In this figure, the results computed by the hybrid FETD-FDTD method and those computed by the ordinary FDTD method for different mesh sizes are compared with the exact result given by the Mie series solution. It can be seen that the results of the hybrid FETD-FDTD method converge rapidly to the exact result as the mesh size is decreased, whereas the results of the ordinary FDTD method converge much more slowly. Indeed, the FDTD results contain significant errors even when an impractical small mesh size of $0.0025 \mu \mathrm{m}$, or roughly $\lambda / 100$, is used. This example shows that highly accurate results can be obtained from a full solution of Maxwell's equations, provided that the proper numerical technique is used for the solution. For this purpose, the hybrid FETD-FDTD method is an attractive numerical technique to use because of its computational efficiency and its ability to model complex shapes ac- curately. Work is currently in progress to increase the computational efficiency and accuracy of the hybrid FETD-FDTD method further, by employing both a higher order FEM and a higher order finite-difference method to reduce the number of nodes per wavelength required to achieve a desired level of accuracy.

\section{USE OF PHENOMENOLOGICAL MODELS AND CONNECTION TO CAD LAYOUT DESIGNS}

\section{A. General Considerations}

At the other end of the detailed simulation spectrum lies an area that has only recently really begun to flourish, namely, the use of fast phenomenological models for predicting the printability of photoresist materials. Here, recent developments will be described that relate improvements in algorithmic development and the use of fast aerial imaging capabilities to the recognition of the need for also including fast phenomenological models to take into account photoresist exposure, baking, and development effects.

Because these phenomenological models ignore many of the detailed physical aspects, such as those discussed in Section II-A, they naturally have inherent limitations. With a clear recognition of such limitations, however, a natural bridge can be formed between the processing world of lithographers and the design world of microchip designers. The much more phenomenological models of the present section purposefully gives up a close physical description in exchange for enormously reduced computational complexity. Consequently, a greater reliance on calibration and a reduced ability of predictability is openly recognized. Nevertheless, the benefits are enormous, as should become apparent shortly.

Several articles have been written in recent years describing the use of fairly simple "resist-bias" models [79]-[86]. These models extend microlithography aerial image simulation capability beyond the simple "threshold energy model" [87], [79], [88] that is often used for obtaining a rough prediction for how an optical mask prints. Here, we will examine general features of these models and try to explain why they achieve good results, as well as discuss their inherent limitations.

Detailed one-dimensional (1-D) and 2-D measurement versus simulation results have been demonstrated with excellent agreement over a range of linewidths [82]-[86]. The 1-D photoresist linewidths predicted with these models correspond to the widths of the bottom edge of long lines and spaces in photoresist, while the 2-D photoresist shapes describe the contour of the bottom edge associated with more general photoresist structures [85], [86]. Examples and general summaries of these agreements, to date, will be discussed in Section IV-B.

Undoubtedly the most important component used in the present set of phenomenological models is the aerial image. Fortunately, over the past eight years or so, several groups have made significant advances in algorithmic approaches to enable rapid calculation of this quantity [24], [25], [89], [81], [88]. Instead of roughly scaling with the square of the area 
for such calculations, as was the case with the original algorithms [23], [90], the fast algorithms available today roughly scale with the area [25]. For a reasonable sized mask region, using the original approach would have been utterly impractical, since the required CPU time would have been measured in orders of years, as opposed to time as measured in minutes or hours with more recent algorithms [25]. Consequently, this new speed has opened up a number of new possibilities, such as enabling information previously only available to lithographers in limited regions to now being practically accessible to microchip designers, process technologists, and manufacturing engineers [91].

Basing fast phenomenological resist-bias models primarily on the aerial image information can be understood to be a reasonable procedure for a number of reasons. In particular, the aerial image contains considerable information regarding the electromagnetic energy distribution being used to expose the photoresist. Of course, it does not contain all the information in this regard, because it is not sufficient to fully predict the propagational and exposure properties of the fields within the photoresist, particularly for high NA projection systems and thicker films [43], [44]. Instead, the aerial image contains only the light intensity information at one plane in the image space of the projection system.

Thus, one immediate consideration is that these models may be tunable to yield good predictions for a given defocus condition, but that without including more detailed information from the incident light field, they should yield lower quality predictions for other defocus values, particularly for thicker resists. Undoubtedly more information from the incident field would help to improve this situation. Calibrating these models to include the defocus has, indeed, recently been found to be helpful [85], [86].

A second point to consider is that the aerial image for any single defocus plane, despite not containing all the physical information about fields in the image space of the projection system, still does contain an enormous amount of information. This fact can be used to good advantage. After all, the rigorous calculation of an aerial image will vary properly with optical parameters, such as NA, aberrations, and partial coherence. Consequently, if simulation predictions based on such information match closely with experiment at one optical setting, then they should be expected to also track fairly well as optical parameters are changed. At the very least, this behavior should hold reasonably well over a limited range, that can be improved with additional calibration methods, as mentioned earlier for the case of defocus. Moreover, one can argue that unless one changes the optical conditions so much as to enter into a regime where a much different basic physical description is required, then changes in the original model settings should be capable of tracking such changes reasonably well. An example of a regime where a different physical description is required occurs when one proceeds from a low or mid-NA situation to a high-NA situation; in the high-NA case, vector aerial image calculations are required and the propagation of the light into the resist becomes much less of a vertical propagation.

A third observation is that the resist bias models that have been developed and reported on so far in the literature

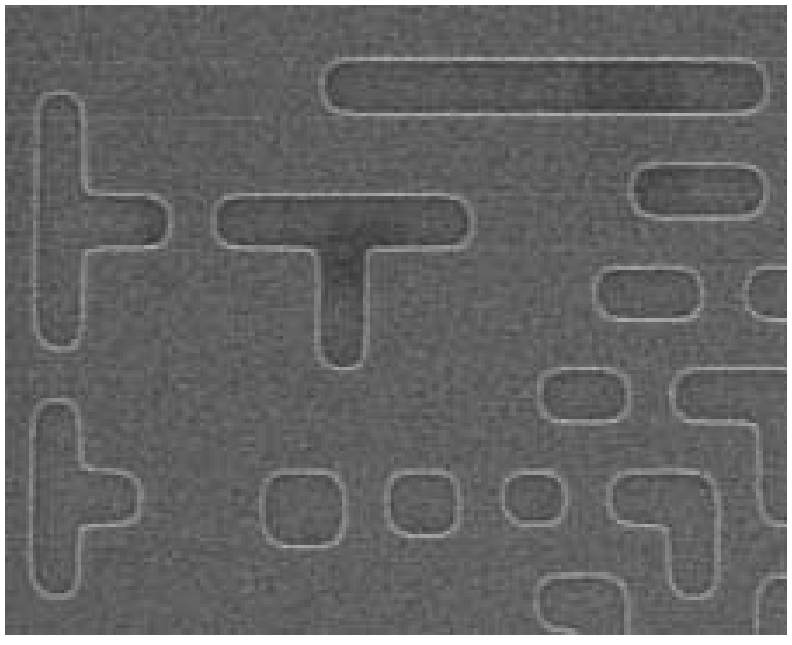

Fig. 5 SEM micrograph of an optical projection mask. The CAD design data for these structures had $0.25 \mu \mathrm{m}$ as the smallest dimension for printing on the wafer, or $1.0 \mu \mathrm{m}$ on the mask $(4 \times$ projection system). In wafer coordinates, the region shown here was roughly $3.0 \mu \mathrm{m}$ by $3.5 \mu \mathrm{m}$. The long bar on the top right was 2 $\mu \mathrm{m}$ long.

[79]-[86] are generally intended to act as a perturbation to the familiar "threshold model" [87], [79], [88]. This threshold model essentially makes the approximation that any point at the top surface of the resist that receives an incident amount of energy above some threshold value will either develop away, or remain, depending on whether the photoresist is positive or negative. The second main assumption in this model is that the energy received below any surface point on the resist is proportional to the aerial image intensity.

Clearly, this model is a very simple one, yet it includes much of the optical information related to diffraction effects involved with imaging a mask. Moreover, there is a key feature associated with this model that can make it fairly powerful, namely, the choice of the contour value of the aerial image that is used to fit measured data; this is the only free parameter in this model. For the 2-D case, this single parameter can be used to force the threshold aerial image prediction to agree with the measured shapes at one point in the set of shapes examined. Used in this way, the aerial image threshold parameter combines all separate controls that actually exist in the photolithography process (such as the exposure, baking, and dissolution times) into a single parameter. By forcing the predicted pattern, such as some critical dimension in Fig. 5, to agree with experiment, the model becomes "tuned" to yield agreement at this point. All other dimensions in the pattern are then determined by the aerial image contour.

Nevertheless, it is certainly important to push beyond this model, as will become clear in Section IV-B. Consequently, a number of parameters that are only indirectly related to the detailed physics have been explored by researchers [79]-[86] to determine how well they can be utilized to gain some of the predictability that exists in more fundamental simulation approaches [14], [17], [27], [30]-[40]. A large number of choices exist, including: 1) information in the optical image 
space, sampled from in and out of focus [81], [92], [85]; 2) information in the focus plane, but along the direction of the normal to the contour predicted from the threshold model [79], [80]; 3) as a "diffused " aerial image [79], [83]-[86], to attempt to account for baking and other "diffusion related" effects; 4) information such as the slope of the aerial image and the maximum peak intensity along a normal to the threshold [82]; or, even 5) information like the curvature of the shape predicted by the threshold model. The extent to how well these models work, and for which resist conditions, will undoubtedly continue to be investigated as tests are made, and as new resist processing is developed. Clearly, none of these approaches can hope to reproduce the more detailed physical models conventionally used [14], [17], [27], [30]-[40], but that is not really the aim; rather, the intent is to find methods that work reasonably well for well-controlled processes, and to use these models, with reasonably rapid calibration methods, to find the necessary fitting parameters for the resist process in question.

\section{B. Results Using Phenomenological Models}

Many industrial groups are presently working on their own proprietary phenomenological models for their own specific resist and etch processes. Here, we report on a specific set of models found to work with good success for the resist processes and dimensions tested. Improvements beyond these can undoubtedly be obtained, but these models clearly show very useful results just as they are, which can indeed bridge gaps presently existing with CAD tools used by designers.

Specifically, here we report on the use of a "diffused aerial image," so as to attempt to model, to some extent, the effects of the diffusion process during post-exposure baking, as well as "... to account for resolution loss due to other sources." 3 We used a fast aerial image simulator, FAIM [24], [25] and a fast means for calculating the diffusion process, namely, where the convolution with a Gaussian was done by taking the inverse Fourier transform of the product of two Fourier transforms. Specifically, the "diffused aerial image" $I_{\mathrm{D}}(\mathbf{x})$ was related to the aerial image intensity $I_{\mathrm{A}}(\mathrm{x})$ by

$$
I_{\mathrm{D}}(\mathrm{x})=\frac{1}{2 \pi L^{2}} \int_{-\infty}^{\infty} \int_{-\infty}^{\infty} d^{2} x^{\prime} \exp \left(\frac{-\left(\mathrm{x}^{\prime}-\mathrm{x}\right)^{2}}{2 L^{2}}\right) I_{\mathrm{A}}\left(\mathrm{x}^{\prime}\right)
$$

where $L$ is the "diffusion length parameter" reported in the next section. To clarify, writing this in the form of a 2-D diffusion equation yields

$$
D\left(\frac{\partial^{2}}{\partial x^{2}}+\frac{\partial^{2}}{\partial y^{2}}\right) I(\mathbf{x}, \mathbf{t})=\frac{\partial}{\partial t} I(\mathbf{x}, \mathbf{t})
$$

which is solved by (3) when $L=\sqrt{2 D t}$.

A second feature of our modeling approach was to characterize the mask making process [93] and to take the imperfections into account due to mask corner rounding (see, e.g., Fig. 5) that typically occur during the mask fabrication process. Here, we note that the IBM mask house that

\footnotetext{
${ }^{3}$ See $[79$, p. 203].
}

we worked with had several mask making processes that could be used, depending on the resolution and turnaround time required for the application. When the mask fabrication process had a high resolution, and the critical dimensions of the mask were not so aggressive, then of course the need for accounting for the mask corner rounding became essentially negligible. Otherwise, the corrected characterized mask was used as input to the aerial image simulator.

Fig. 5 shows a scanning electron microscope (SEM) micrograph of a mask made with the Etec Systems' ALTA laser beam mask writing tool. This mask was used for the study described here. As can be seen in Fig. 5, although the mask is of high quality, there is a significant rounding of corners that is inherent to this mask making process. The precise SEM measurements of the mask were used in generating our predicted printed shapes, enabling a more detailed calibration of our model to the measured printed images. We note that there have been several mask processes taken into account in the course of our studies. As described elsewhere [93], the mask processes can vary considerably from one semiconductor technology to another, and from one mask level to another within a given technology. Typically this choice is intentional, since there is a recognized tradeoff in the mask accuracy required for a particular semiconductor technology and process level in question, versus the cost and turnaround time in fabricating the mask.

Third, there was one other "free parameter," besides the: 1) threshold (i.e., contour chosen in the final "diffused image") and 2) "diffusion length" that was calibrated in this modeling work; namely, 3) the defocus value. It is well known that the absolute defocus value in microlithography is a difficult value to ascertain, particularly within a few tenths of a micrometer; differences from one setting to another are very well controlled, but the absolute value, in relation to the Gaussian image plane, is a much more difficult quantity to know precisely. Moreover, one can certainly legitimately ask, from a modeling standpoint, what is the correct value of defocus to use when calibrating this phenomenological model to fit a set of shapes, particularly when considering photoresist films on the order of a micrometer in thickness.

We chose to fit our model to the measured photoresist shapes described in the next section at not just one focus setting, but over a range of focus values, where the differences between focus conditions were determined by controlling the wafer stage displacements, but the single "absolute defocus value" for the family of data was used as a fitting parameter in the model. We found, not surprisingly in hindsight, that this parameter had a similar effect to changes in the diffusion length $L$ in the model, undoubtedly because both parameters act, roughly speaking, to smear the resulting image.

Nine of the shapes in Fig. 5 were selected for calibration purposes, namely, all but those in the bottom right of the figure. The calibration shapes included the three "T" shapes shown, as well as six of the seven "bar" shapes. For our choice of photoresist, stepper parameters, and resist processing conditions, the smallest bar in Fig. 5 was poorly resolved and exhibited residual resist. For this reason, it was omitted from this study. However, the calibration shapes 


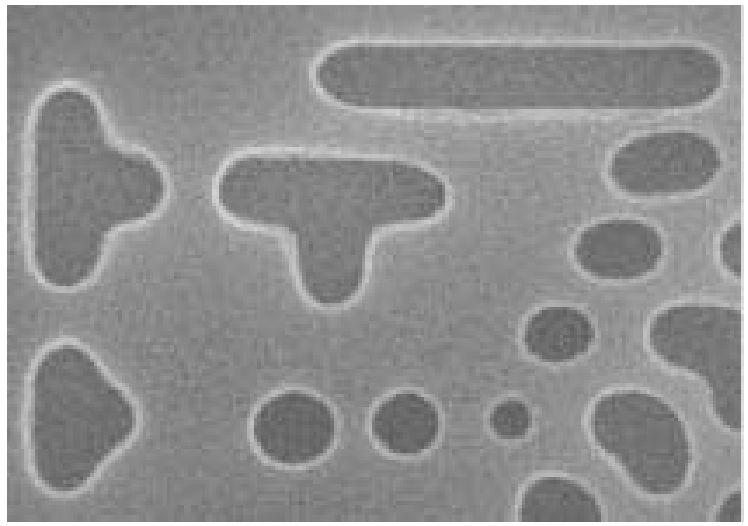

Fig. 6 SEM of UV2HS photoresist structures, corresponding to the mask pattern shown in Fig. 5. The nominal focus setting used here was $0.0 \mu \mathrm{m}$ from the Gaussian image plane.

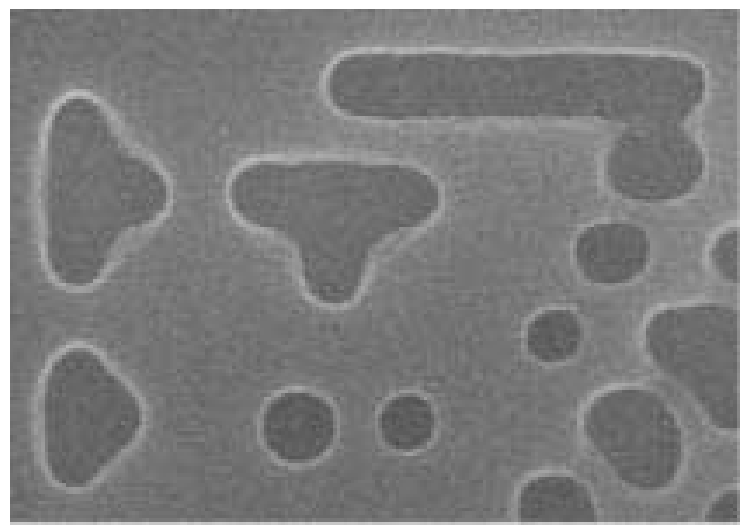

Fig. 7 SEM of UV2HS photoresist structures, corresponding to the mask pattern shown in Fig. 5. The nominal focus setting used here was $0.75 \mu \mathrm{m}$ from the Gaussian image plane. As can be seen, the smallest structure in the bottom right of Fig. 5 does not appear here, as it does not print at this large defocus condition.

included the smallest dimensions used in the lithographic process being characterized. The "T" shapes in Fig. 5 ensured that "inside" and "outside" corners were included in the calibration, as well as horizontal and vertical shapes. The smaller "contact" bars ensured that shapes with low maximum aerial image intensity and small slopes were included. These nine shapes, with $0.25-\mu \mathrm{m}$ minimum critical dimensions, along with seven similar "T" and "bar" shapes with $0.35-\mu \mathrm{m}$ critical dimensions, were used to calibrate a $0.25-\mu \mathrm{m}$ UV2HS lithographic process.

Figs. 6 and 7 show top-down SEMs of the printed shapes in UV2HS photoresist, using the mask in Fig. 5. Figs. 6 and 7 correspond, respectively, to nominal focus settings at 0.0 and $0.75 \mu \mathrm{m}$ from the Gaussian image plane. As can be seen, the smallest structure in the bottom right of Fig. 5 does not print in Fig. 7, due to the large defocus value. A defocus setting of $0.75 \mu \mathrm{m}$ far exceeds the allowed process window in manufacturing (the process window is more typically within $\pm 0.3 \mu \mathrm{m}$ ), but serves as an excellent test of the present phenomenological model.

After 2-D SEM calibration procedures [85], [86] were carried out, detailed comparisons were made between simulation and experiment. Figs. 8 and 9 show contour predic-

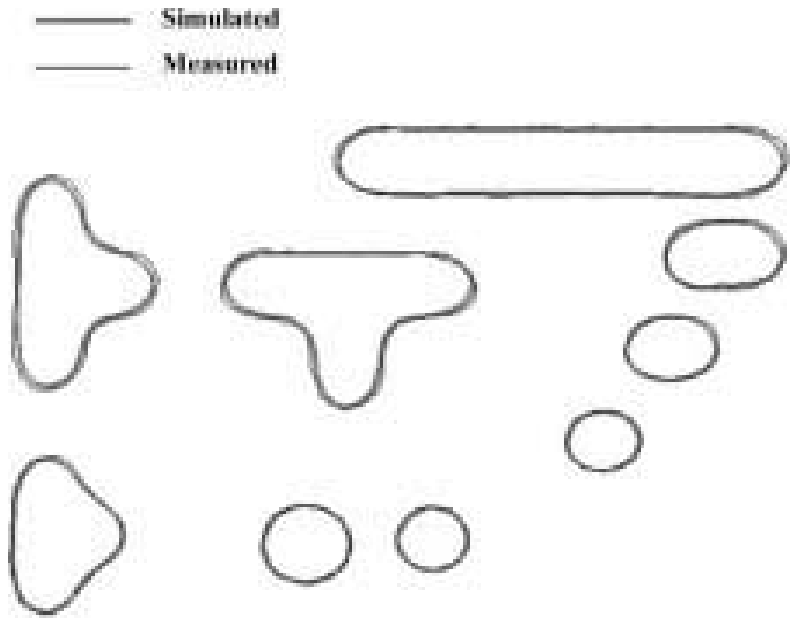

Fig. 8 Superposition of simulation and measurement for the in-focus case of nine of the photoresist shapes in Fig. 5.
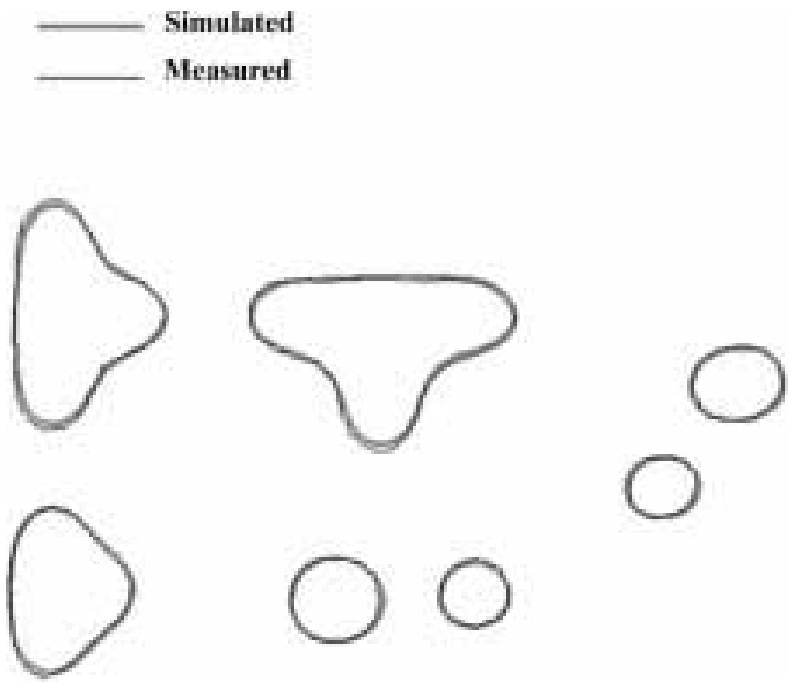

Fig. 9 Comparison between SEM measurement of UV2HS structures and simulation at $0.75 \mu \mathrm{m}$ defocus.

tions from simulation, using the phenomenological model discussed here, versus SEM edge detection of the shapes in Figs. 6 and 7. As can be seen, the agreement is quite good. Far more detailed characterization of the agreement was used, however, as shown in Figs. 10 and 11. Plots such as these were used to represent and analyze the distribution of distances and directions between corresponding simulated and measured points. The lengths of the vectors in Figs. 10 and 11 are scaled to the magnitude of the differences between simulation and measurement, thereby enabling easy identification of the regions of largest deviation. Probing into the source of the regions of large deviations often resulted in the identification of a mask fabrication defect, a poor SEM signal, or a poor quality resist edge.

The upper left-hand corner of Fig. 10 contains a summary of the distribution of vector lengths. For example, the "100>0 nm," "87> $>5 \mathrm{~nm}$," and "72> $10 \mathrm{~nm}$ " specifications indicate that 100 vectors were contained in the plot, $(100-87)=13$ of them with lengths less than $5 \mathrm{~nm}$ $(87-72)=15$ of them with lengths between 5 and $10 \mathrm{~nm}$, 


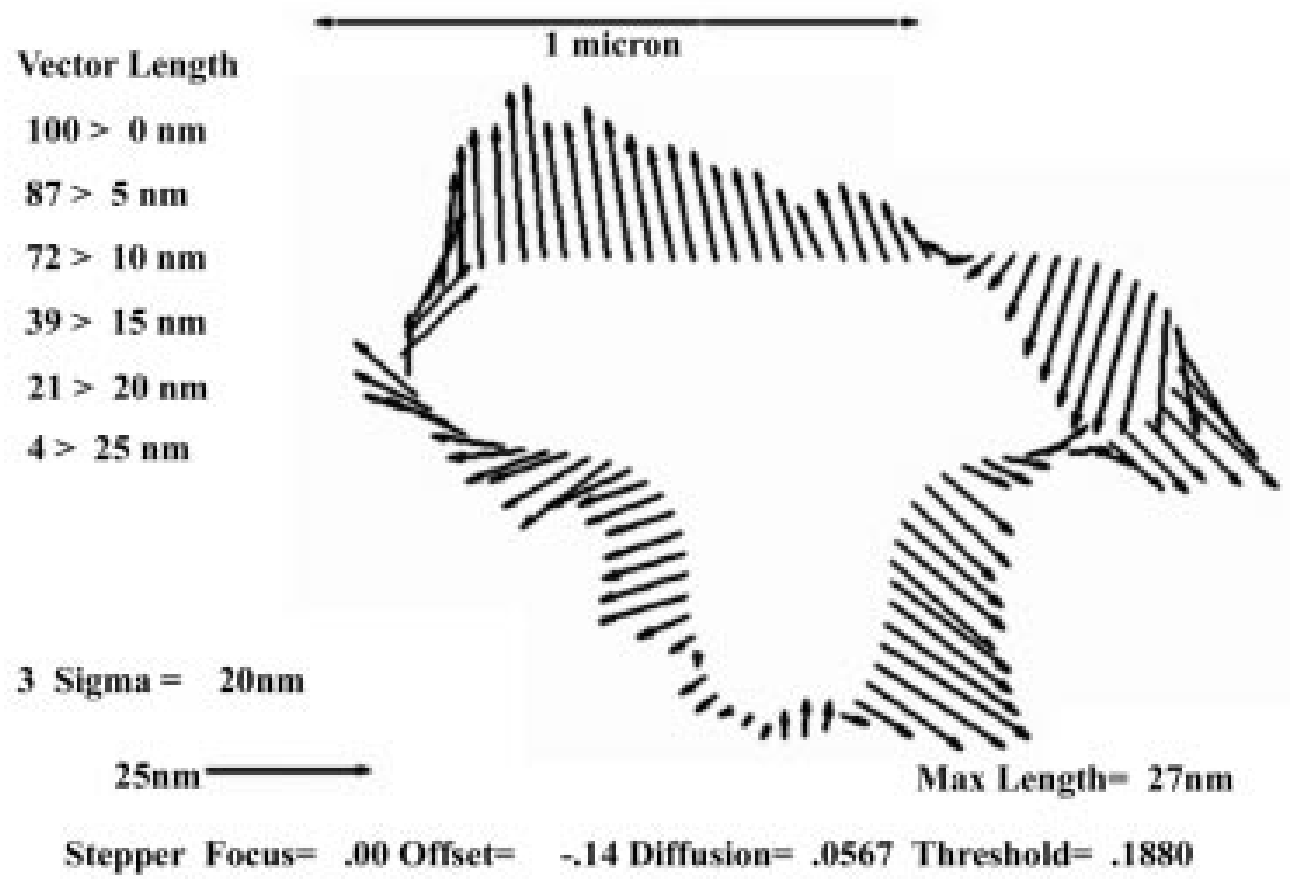

Fig. 10 Vector plot of the deviations between simulation and SEM measurements corresponding to one of the UV2HS printed "T" shapes in Fig. 5.

etc. The "3 Sigma" designation in the lower left indicates the value of $3-\sigma$ standard deviations for the data. The maximum vector length can be found in the lower right of the plot, which here was $27 \mathrm{~nm}$.

Fig. 11 demonstrates the results when the plot is expanded to include a larger number of shapes. Here, 4500 measurements of the difference between simulation and experiment are displayed. Consequently, individual vectors cannot be distinguished. However, the width of the band provides helpful information. Moreover, displaying the distribution of vector lengths in "bins," with each in a different color, aids analysis considerably.

Using the simple, but fast phenomenological model as described here, at our present level of sophistication in applying these methods, we have typically found that after proper calibration, the shapes of photoresist images can be predicted to within a 3- $\sigma$ variation equal to $15 \mathrm{~nm}$, for all points on the 2-D shapes. This value applies to 2-D photoresist shapes exposed with 248-nm photolithography for $0.25 \mu \mathrm{m}$ critical dimension images at zero defocus. Evaluation of samples exposed at $0.5 \mu \mathrm{m}$ defocus typically increases this $3-\sigma$ variation to about $40 \mathrm{~nm}$ for our optical exposure conditions of 0.5 for the NA and 0.6 for the circular partial coherence parameter.

By carefully characterizing a stable resist process, where the film type and thickness, baking cycle, and dissolution step are all fixed, then appropriate phenomenological parameters can be found that do an excellent job of predicting the final printed shape patterns. Changing critical dimensions significantly may require the calibration of new parameters, or extensions of these models, if new physical effects then become important, such as nonlinear diffusion effects. Nevertheless, the method seems reasonably clear, that by exam- ining a range of shapes and sizes that will typically be produced in a circuit pattern, and where minimum critical sizes exist, as is always the case in present microlithography situations, then fast, accurate phenomenological models can be imposed that will greatly aid in characterizing shapes over an entire circuit pattern.

As will be discussed in Section V, by then adding additional shapes, or "serifs," to the corners and edges of patterns, as well as by simply biasing edges, then final printed shapes can be printed much closer to the actual intended designs. Such "proximity" corrections require highly sophisticated optimization procedures, however, as discussed in Section V.

\section{Connecting with CAD Layout Methods}

Present practices in circuit layouts nearly exclusively use the rectilinear patterns that give rise to the shapes in Fig. 5, despite the now well-known differences that exist between these patterns, the ones on the mask (see Fig. 5), and the ones in the actual photoresist patterns on the wafer (see Fig. 6). Upon visually seeing these differences, undoubtedly one's immediate reaction dwells on why these differences are not better taken into account in very large scale integration (VLSI) CAD design. After all, such differences in shapes will clearly have impacts on the intersected contact areas between levels, which affects allowable current density levels. Moreover, shapes of other structures will affect critical signal delays, due to capacitance and resistance changes.

Now, it should be noted that at the single device level, these differences in shapes are certainly well known and usually carefully taken into account. They have to be, since 


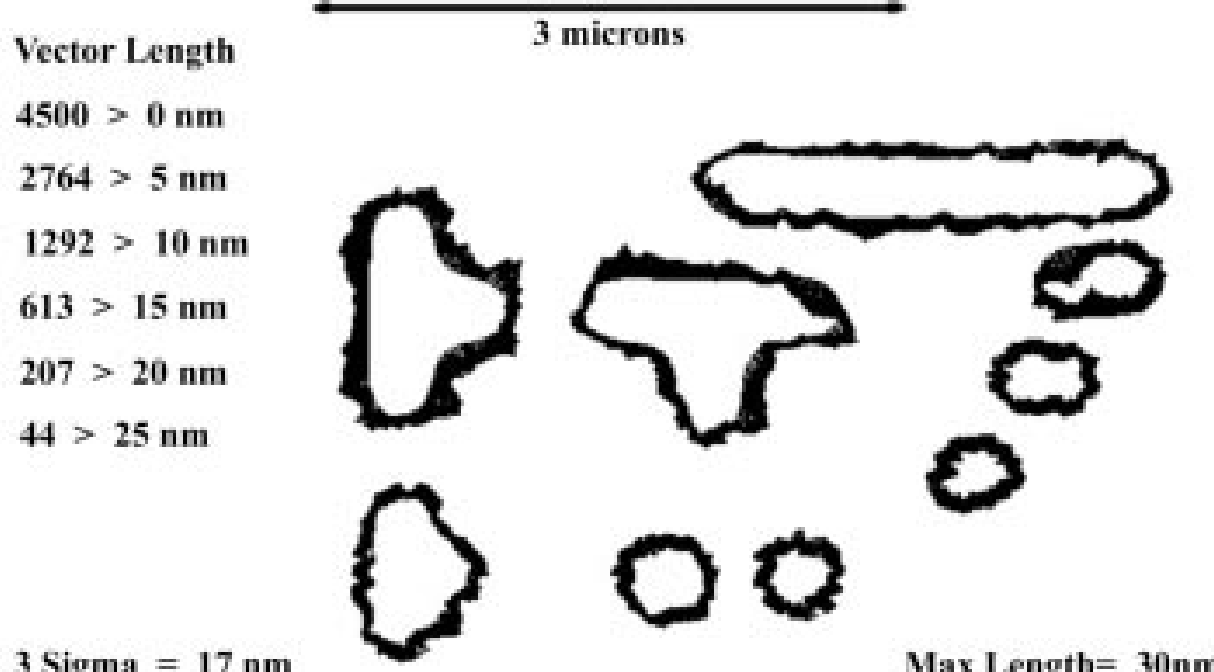

3 Sigma $=17 \mathrm{~nm} \quad$ Max Length $=30 \mathrm{~nm}$

$25 \mathrm{~nm} \rightarrow$

Stepper Focus $=.25$ Offset= -.14 Diffusion= .0567 Threshold $=.1880$

Fig. 11 Vector plot, as in Fig. 10, but containing the deviations for all nine of the structures in

Fig. 6, between the SEM detected edge of the resist structure and the simulated predictions.

Displaying this plot in color, with each set of vectors having a separate color, aids in distinguishing

between the regions.

changes in gate shapes, storage trench sizes, etc., all have to be carefully controlled to obtain the correct device behavior. However, when putting many devices together, and when accounting for proximity effects of nearby devices on pattern shape alterations, the changes in shapes are only now beginning to be carefully implemented at the detailed level as presented here.

As discussed in [91], several reasons exist for this divide. Certainly, the present high speed algorithms have only relatively recently become available, and the new well-calibrated phenomenological models for 2-D shapes is yet even more recent. Moreover, and perhaps more importantly, the data volumes rise enormously with this better characterization. Whereas a single rectilinear shape would only previously have required four sets of $x$ and $y$ coordinates, now far more points would be required to characterize the curved shapes. Lastly, current practices and divisions in practice between designers, technologists, and manufacturing engineers will require changes to help merge the knowledge and usage from one area to another. The present practice of relaying and restricting deviations from the rectilinear patterns in circuit design is done via a list of "design rules" that encode such things as the smallest contact area, proximity relations, etc. Properly merging the additional information in lithography simulation into design tools in the most practical manner will require dedicated efforts, acceptance, and training within the industry. ${ }^{4}$

\footnotetext{
${ }^{4}$ Examples of overlaid CAD shapes, simulation prediction, and comparison with experiment, for multiple levels, are shown in [91].
}

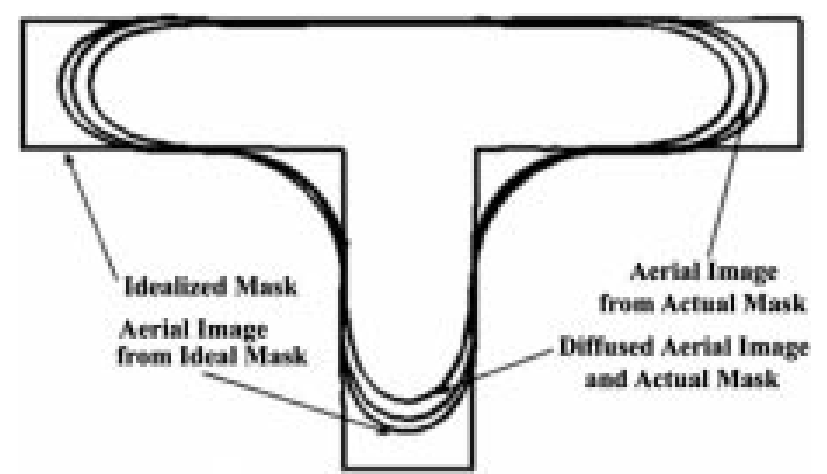

Fig. 12 Superposition of predictions from a threshold aerial image model (outside curve), versus the threshold aerial image model with mask corrections (middle curve), versus a diffused aerial image threshold model, with mask corrections (inner curve). The inner curve matches experimental data closely.

To illustrate more explicitly the differences between the CAD rectilinear shapes and three successive levels of approximation in the phenomenological simulation models, Fig. 12 shows the comparisons laid on top of each other for the "T" shape in Fig. 10. As can be seen, the first order-correction due to a threshold-energy aerial image model is significantly different from the rectilinear CAD design. Upon taking the corner rounding on the optical mask into account (Fig. 5 shows typical mask corner rounding), one obtains the next improvement in prediction. The most inner curve in Fig. 12 shows the prediction using the corner rounding plus diffused aerial image. This curve lies virtually 
on top of the SEM measurement, at the magnification showed in this figure, and as displayed more explicitly in Figs. 8 and 10.

\section{OPTIMIZATION OF RESOLUTION ENHANCEMENT TECHNIQUES}

A number of novel optical enhancement resolution techniques have been introduced and explored with considerable interest in microlithography during the past decade, including ${ }^{5}$ proximity correction methods (serifs and auxiliary support structures) [94]-[99], phase-shift masks [51], [100], off-axis illumination methods [52], [53], pupil filters, and focus-modulation methods [101]. These techniques have enabled the use of optical lithographic methods to extend far beyond what most researchers had predicted a decade ago. About that time, most researchers felt the smallest dimensions that could be produced with projection printing were roughly equal to the wavelength of the illuminating radiation. However, present production chips already have minimum dimensions equal to $0.18 \mu \mathrm{m}$ using $0.248-\mu \mathrm{m}$ wavelength light, thereby clearly pushing well beyond the previously assumed limit. Moreover, there is considerable impetus that yet further improvements are achievable, namely, to sub-half wavelength feature size printability [53].

What has become very apparent is that by making the optical mask considerably different than what one really needs to print, then far greater resolution capability is achievable than what would be obtained from a simple application of Rayleigh's criteria in optics [20]. Rayleigh's criteria provides a rough estimate of the resolution that is achievable if one attempts to make the image of an object be very similar to the object in optics. However, if one is willing to make the object considerably different than the image, such as by including "subresolution" elements that are not intended to print themselves, but that are intended to aid in the printability of another object, then Rayleigh's criteria no longer applies.

An example of this effect is shown in Figs. 13 and 14. Fig. 13 shows a gate pattern that might be used in making an SRAM. If the minimum feature size is close to the wavelength of the illuminating light, then the printed features will be significantly different from the intended design, due in part to diffraction effects. In particular, the ends of the lines will be shortened, and each corner will be significantly rounded. However, by added "serifs," line extensions, and linewidth biases, such as those shown in Fig. 14, then much improved printability can be obtained.

An even more dramatic effect can often be obtained by including changes in transmission in the object so as to introduce destructive and constructive interference components in the path of the transmitted light [51], [100]. These elements act to "phase shift" the transmitted light. Now, the mask can become even more significantly different from the intended printed pattern, with yet greater potential for improving printability. Such results have been well documented in the literature, from as far back as 1982 [100]. However, the

\footnotetext{
${ }^{5}$ Please see the numerous references cited in the following articles as well.
}

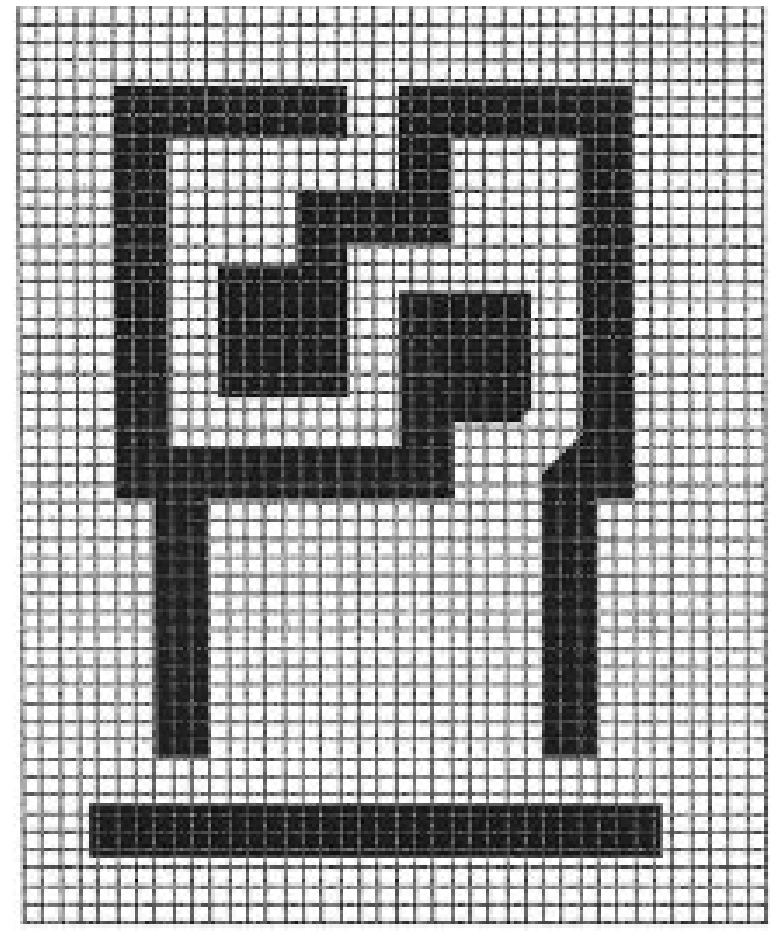

Fig. 13 SRAM CAD design for gate level of MOSFET structures.

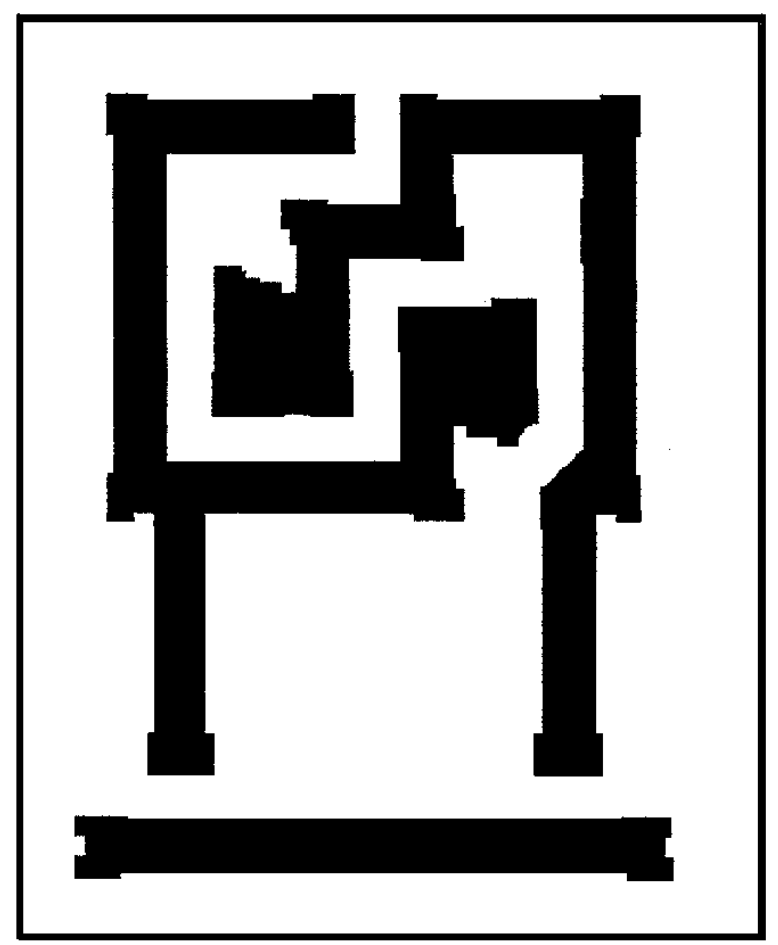

Fig. 14 SRAM CAD design with subresolution proximity corrections consisting of "serifs," edge biases, and line extensions to enable the final printed photoresist structure print closer to the intended design structure shown in Fig. 5.

difficulty in making reliable phase-shift mask structures for a wide range of pattern shapes and sizes, all with adequate process windows (i.e., where the images can be reliably produced despite unavoidable variations in focusing and exposure conditions), has been a major difficulty in realizing this full potential. 
Likewise, changing how these masks are illuminated, such as by controlling the degree of partial coherency of the incident light and the direction of incidence, and possibly coupling this change in illumination with phase shifting methods, can provide significant additional benefits [52], [98]. Each of these methods can improve the imaging and projection printing of microcircuit designs, but due to their nonlinear nature, it is not easy to a priori predict when they compete against each other with degraded results. In other words, a potentially improved methodology can actually reduce printability if improperly placed. For this reason, the optimization of the availability of these numerous effects has become a very important issue in optical microlithography. The optimization must take into account the restrictions imposed on the manufacturing process of the masks and the exposure of the wafers. Because the optimization is design dependent, then the problem is far from trivial.

Hence, there are a number of competing complicating effects. First, of course, to optimize adequately, requires a costfunction that reasonably reflects what one wants to achieve in practice. Second, computing complexities can restrict the full usefulness of such a definition. Third, engineering flexibilities on what can reasonably be altered in a cost-efficient and time constrained manner must be taken into account. Active research along these lines is a major part of present microlithography simulation efforts.

Reference [53] describes an optimization approach that combines proximity correction effects using serif-like structures, as in Fig. 14, with off-axis illumination aperture optimization, with an attenuated phase shift mask structure [102]. (The attenuated PSM structure is generally considered a much easier mask to construct than most other PSM forms). The initial optimization scheme employed was a modified conjugate-gradient with a Kamon illuminator [103], [104] shape as the initial condition, with the smallest printable slope of the aerial image as the initial cost function. An improvement in the minimum contrast was obtained with a systematic march that depended on the various derivatives in "illuminator space." Optimization was also performed at the limiting end value of the desired defocus range, thereby improving printability throughout the entire defocus budget.

Combining algorithmically this illuminator optimization with mask proximity corrections enabled necessary corrections to aggressive SRAM mask designs with feature sizes as small as $140 \mathrm{~nm}$ when employing 248-nm illumination. Fig. 15 illustrates the predicted contour of the printed shapes. Fig. 16 shows a contour plot of the associated aerial image associated with this mask structure. As one can see, the contrast associated with this imaging is very good.

Further work continues on constructing such masks and experimenting with the best methodology for taking CAD designs and turning them into manufacturable masks and photoresist patterns. Besides the mathematical problems of optimization in reasonable execution times, as referred to here, other key concerns are properly folding these methods into the usual practice of lithography and microchip design, where groundrule pattern layout specification, groundrule

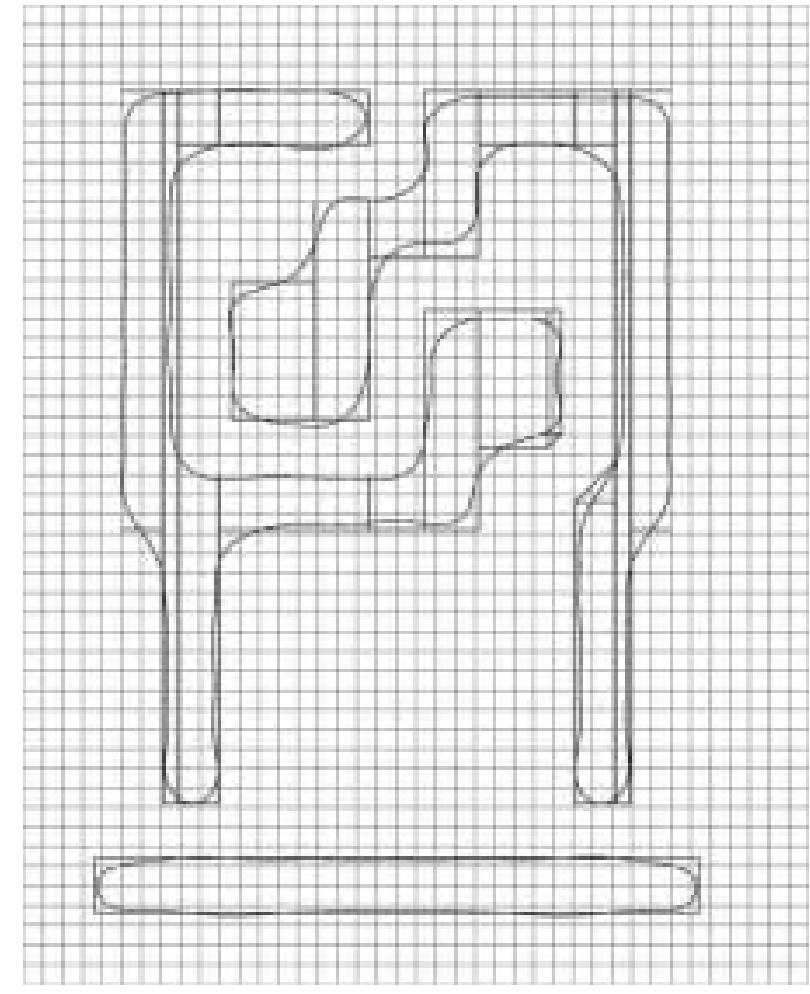

Fig. 15 Predicted contour of printed photoresist shape after optimizing the illumination conditions, the serif structures (as in Fig. 14), and using an attenuated PSM. The smallest feature size here is $140 \mathrm{~nm}$; the wavelength is $248 \mathrm{~nm}$.

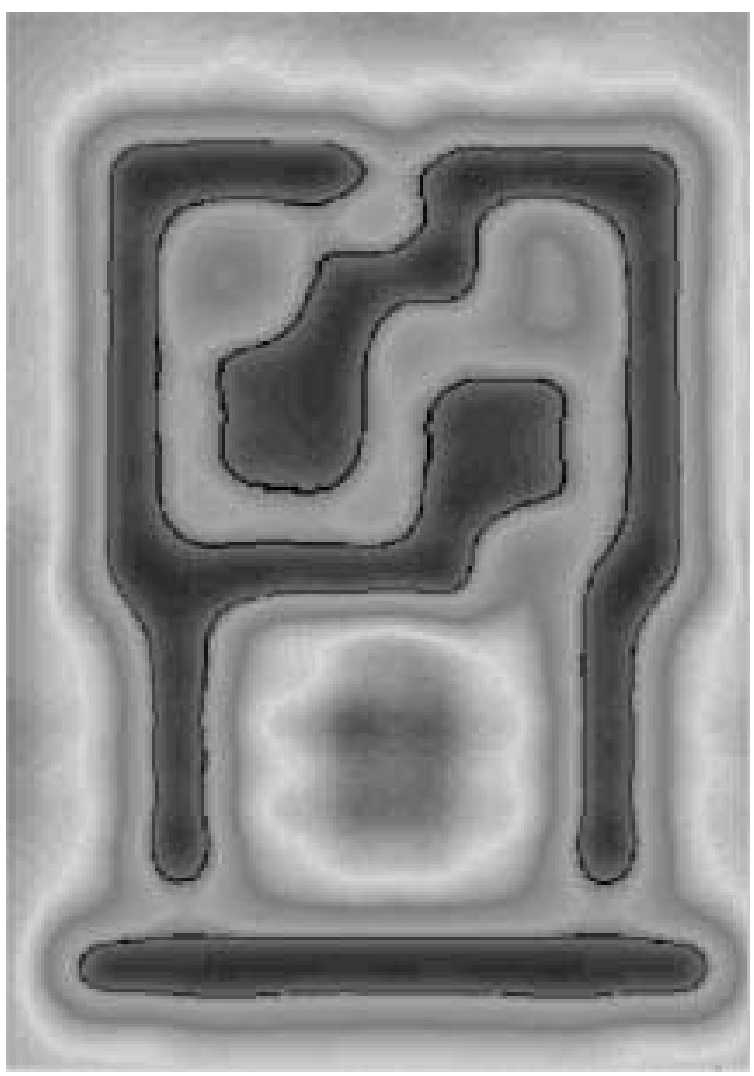

Fig. 16 Contour plot of the calculated aerial image corresponding to the optimized conditions associated with Fig. 15. 
checking, mask construction, mask writing, and mask repair, are all key concerns.

\section{CONCLUDing REMARKS}

This paper first surveyed the early development of microlithography simulation, then gave a brief summary of work done since that time. Three areas of more recent development were next described, namely: 1) a detailed solution of Maxwell's equations for propagating light through a complex reticle structure, such as would be used in phase-shift mask technology; 2) phenomenological models that describe resist and etch development processes; and 3) the optimization of microlithographic resolution enhancement effects. Without question, there are other very important areas that could be discussed, such as a more recent detailed probing into photoresist processing at the molecular level [105], new photoresist chemistries, work done on alignment structures, surface imaging methods, investigations into improved process windows, modeling of film deposition, and imaging and exposure using new sources of radiation. However, our aim here was to give a brief review, then show three very representative problems of key interest, including one very fundamental physical area (Section III) and one very phenomenological area (Section IV). Both approaches can be extremely useful, although usually not to the same group of technologists. For example, a detailed photoresist development simulator is of more use to a lithographer developing a photoresist process, where the best combination of film type, film thickness, and exposure and baking conditions is not yet known. In contrast, the phenomenological resist bias models described in Section IV are of more use to a mask designer and perhaps a manufacturing engineer and a circuit designer. These models are only applicable once the lithographic processes are well established and controlled, as the models cannot make accurate predictions on changes in film thickness, for example. Instead, such models rely heavily on calibration methods.

As can be seen, the simulation of photoresist processing has numerous ties with other areas of engineering. We anticipate that further advances in fundamental understanding, computational methods, computer hardware, and integration of lithography simulation methods into other areas of engineering, particularly in both the CAD design areas and manufacturing areas, will continue. This capturing and testing of knowledge by simulation methods, via close comparisons with experimental efforts, is essential for continued strong developments in microlithography, as well as for considerations into the area of nanotechnology.

As can be seen, the simulation of photoresist processing has numerous ties with other areas of engineering. We anticipate that further advances in fundamental understanding, computational methods, computer hardware, and integration of lithography simulation methods into both CAD and manufacturing areas will continue. Simulation methods that are closely coupled with experimental testing will remain essential for the future development of microlithography, as well as for the explorations of alternative technologies in the design and manufacturing of microelectronic circuits, and progression into the nanotechnology arena.

\section{REFERENCES}

[1] L. F. Thompson, C. G. Willson, and M. J. Bowden, Eds., Introduction to Microlithography, 2 ed. Washington, D. C: Amer. Chemical Soc., 1994.

[2] S. Wolf and R. N. Tauber, "Silicon processing for the VLSI era," in Process Technology. Sunset Beach, CA: Lattice Press, 1986, vol. 1.

[3] S. A. Campbell, The Science and Engineering of Microelectronic Fabrication. New York: Oxford Univ. Press, 1996.

[4] D. C. Cole, E. M. Buturla, and S. S. Furkay et al., "The use of simulation in semiconductor technology development," Solid State Electron., vol. 33, pp. 591-623, 1990.

[5] G. E. Moore, "Cramming more components onto integrated circuits," Electronics, vol. 38, pp. 114-117, 1965.

[6] - "Progress in digital integrated electronics," IEEE IEDM Tech. Dig., pp. 11-13, 1975.

[7] , "Lithography and the future of Moore's law," in Proc. SPIE Optical/Laser Microlithography VIII, vol. 2440, T. A. Brunner, Ed., 1995, pp. 2-17.

[8] J. H. Bruning, "Optical lithography - thirty years and three orders of magnitude," in Proc. SPIE Optical Microlithography X, vol. 3051, G. E. Fuller, Ed., 1997, pp. 14-27.

[9] F. H. Dill, "Optical lithography," IEEE Trans. Electron Devices, vol. ED-22, pp. 440-444, July 1975.

[10] F. H. Dill, W. P. Hornberger, P. S. Hauge, and J. M. Shaw, "Characterization of positive resist," IEEE Trans. Electron Devices, vol. ED-22, pp. 445-452, July 1975.

[11] K. Konnerth and F. H. Dill, "In-situ measurement of dielectric thickness during etching or developing processes," IEEE Trans. Electron Devices, vol. ED-22, pp. 452-456, July 1975.

[12] F. H. Dill, A. R. Neureuther, J. A. Tuttle, and E. J. Walker, "Modeling projection printing of positive photoresists," IEEE Trans. Electron Devices, vol. ED-22, pp. 456-464, July 1975.

[13] E. J. Walker, "Reduction of photoresist standing-wave effects by post-exposure bake," IEEE Trans. Electron Devices, vol. ED-22, pp. 464-466, July 1975.

[14] W. G. Oldham, S. N. Nandgaonkar, A. R. Neureuther, and M. M. O'Toole, "A general simulator for VLSI lithography and etching processes. Part 1-application to projection lithography," IEEE Trans. Electron Devices, vol. ED-26, pp. 717-722, Apr. 1979.

[15] W. G. Oldham, A. R. Neureuther, C. Sung, J. L. Reynolds, and S. N. Nandgaonkar, "A general simulator for VLSI lithography and etching processes. Part II-application to deposition and etching," IEEE Trans. Electron Devices, vol. ED-27, pp. 1455-1459, Aug. 1980.

[16] C. A. Mack, "Prolith: A comprehensive optical lithography model," in Proc. SPIE Optical Microlithography IV, vol. 538, H. L. Stover, Ed., 1985, pp. 207-220.

[17] - Inside Prolith: A Comprehensive Guide to Optical Lithography Simulation: FINLE Technologies, Inc., 1997.

[18] J. D. Jackson, Classical Electrodynamics. New York: Wiley, 1998.

[19] D. C. Cole, "Cross-term conservation relationships for electromagnetic energy, linear momentum, and angular momentum," Found. Phys., vol. 29, pp. 1673-1693, 1999.

[20] M. Born and E. Wolf, Principles of Optics, 6th ed. Cambridge, U.K.: Cambridge Univ. Press, 1998.

[21] J. W. Goodman, Introduction to Fourier Optics, 2nd ed. New York: McGraw-Hill, 1996.

[22] - Statistical Optics. New York: Wiley, 1985.

[23] H. H. Hopkins, "On the diffraction theory of optical images," Proc. R. Soc. Lond. A, vol. 217, pp. 408-432, 1953.

[24] D. C. Cole, E. Barouch, U. Hollerbach, and S. A. Orszag, "Derivation and simulation of higher numerical aperture scalar aerial images," Jpn. J. Appl. Phys., vol. 31, pp. 4110-4119, 1992.

[25] E. Barouch, D. C. Cole, U. Hollerbach, and S. A. Orszag, "Vector aerial image with off-axis illumination," in Proc. SPIE Optical/Laser Microlithography VI, vol. 1927, J. D. Cuthbert, Ed., 1993, pp. 686-708.

[26] O. S. Heavens, Optical Properties of Solid Films. New York: Dover, 1991. 
[27] E. Barouch, U. Hollerbach, S. A. Orszag, and J. W. Cahn, "Numerical simulation of submicron photolithographic processing," J. Sci. Comput., vol. 6, pp. 229-250, 1991.

[28] R. E. Jewett, P. I. Hagouel, A. R. Neureuther, and T. Van Duzer, "Line-profile resist development simulation techniques," Polym. Eng. Sci., vol. 17, pp. 381-384, June 1977.

[29] P. I. Hagouel, "X-ray lithography fabrication of blazed diffraction gratings,” Ph.D., University of California at Berkeley, 1976.

[30] E. Barouch, B. D. Bradie, and S. V. Babu, "Resist development described by least action principle-line profile prediction," J. Vac. Sci. Technol. B, vol. 6, pp. 2234-2237, 1989.

[31] E. Barouch, B. Bradie, and S. V. Babu, "Three dimensional profile simulation for positive photoresists," in Proc. SPIE Advances in Resist Technology and Processing VI, vol. 1086, E. Reichmanis, Ed., 1989, pp. 495-501.

[32] B. Bradie, "Comprehensive simulation of photolithographic processes in two and three dimensions," Ph.D. dissertation, Clarkson University, 1990.

[33] E. Barouch, B. Bradie, G. Karniadakis, and S. Orszag, "Comprehensive 3D simulator with nonplanar substrates," in Proc. SPIE Optical/Laser Microlithography III, vol. 1264, V. Pol, Ed., 1990, pp. 334-342.

[34] E. Barouch, U. Hollerbach, S. Orszag, B. Bradie, and M. Peckerar, "Process latitudes in projection printing," in Proc. SPIE ElectronBeam, X-Ray, and Ion-Beam Submicrometer Lithographies for Manufacturing, vol. 1465, M. C. Peckerar, Ed., 1991, pp. 254-262.

[35] E. Barouch, B. Bradie, U. Hollerbach, S. A. Orszag, and M. Peckerar, "Modeling process latitude in UV projection lithography," IEEE Electron Device Lett., vol. 12, pp. 513-515, 1991.

[36] E. Barouch, U. Hollerbach, S. A. Orszag, C. R. Szmanda, and J. W. Thackeray, "Simulations of bar printing over a MOSFET device using I-line and deep-UV resists," in Proc. SPIE Optical/Laser Microlithography IV, vol. 1463, V. Pol, Ed., 1991, pp. 464-474.

[37] K. K. H. Toh, "Algorithms for three-dimensional simulation of photoresist development," Ph.D. dissertation, Univ. California, Berkeley, 1990.

[38] K. K. H. Toh, A. R. Neureuther, and E. W. Scheckler, "Algorithms for simulation of three-dimensional etching," IEEE Trans. Computer-Aided Design, vol. CAD-13, pp. 616-624, 1994.

[39] J. J. Helmsen, "A comparison of three dimensional photolithography simulators,” Ph.D. dissertation, Univ. California, Berkeley, 1994.

[40] S. Brainerd, D. Bernard, J. Rey, J. Li, Y. Granik, and V. Boksha, "Sub-half micron contact window design with 3D photolithography simulator," in Proc. SPIE Optical Microlithography X, vol. 3051, G. E. Fuller, Ed., 1996, pp. 552-566.

[41] D. Adalsteinsson and J. A. Sethian, "A level set approach to a unified model for etching, deposition, and lithography. 1. algorithms and 2-dimensional simulations," J. Comput. Phys., vol. 120, pp. 128-144, 1995.

[42] — " "A level set approach to a unified model for etching, deposition, and lithography. 2. 3-dimensional simulations," J. Comput. Phys., vol. 122, pp. 348-366, 1995.

[43] M. S. Yeung, "Modeling high numerical aperture optical lithography,” in Proc. SPIE Optical/Laser Microlithography, vol. 922, B. J. Lin, Ed., 1988, pp. 149-167.

[44] D. A. Bernard and H. P. Urbach, "Thin-film interference effects in photolithography for finite numerical apertures," J. Opt. Soc. Amer. A, vol. 8, no. 1, pp. 123-133, 1991.

[45] B. W. Smith, D. G. Flagello, J. R. Summa, and L. F. Fuller, "Comparison of scalar and vector diffraction modeling for deep-UV lithography," in Proc. SPIE Optical/Laser Microlithography VI, vol. 1927, J. D. Cuthbert, Ed., 1993, pp. 847-857.

[46] D. G. Flagello and T. D. Milster, "High-numerical-aperture effects in photoresist," Appl. Opt., vol. 36, pp. 8944-8951, 1997.

[47] E. Wolf, "Electromagnetic diffraction in optical systems: I. an integral representation of the image field," Proc. R. Soc. A, vol. 253, pp. 349-357, 1959.

[48] B. Richards and E. Wolf, "Electromagnetic diffraction in optical systems: II., structure of the image field in an aplanatic system," Proc. R. Soc. A, vol. 253, p. 358, 1959.

[49] C. A. Spence, R. A. Ferguson, A. R. Neureuther, and W. G. Oldham, "Characterization and modeling of materials for photolithographic simulation,” Solid State Electron., vol. 33, pp. 625-638, 1990.
[50] J. L. Jessop, S. N. Goldie, A. B. Scranton, G. J. Blanchard, B. Rangarajan, L. Capodieci, R. Subramanian, and M. K. Templeton, "Characterizing acid mobility in chemically amplified resists via spectroscopic methods," in Proc. SPIE Advances in Resist Technology and Processing XVI, vol. 3678, W. E. Conley, Ed., 1999, pp. 914-922.

[51] B. J. Lin, "Phase-shifting and other challenges in optical mask technology," in Proc. SPIE 10th Annu. Symp. Microlithography, vol. 1496, J. N. Wiley, Ed., 1990, pp. 54-79.

[52] — - "Off-axis illumination-working principles and comparison with alternating phase-shifting masks," in Proc. SPIE Optical/Laser Microlithography VI, vol. 1927, J. D. Cuthbert, Ed., 1993, pp. 89-100.

[53] E. Barouch, S. L. Knodle, S. A. Orszag, and M. S. Yeung, "Illuminator optimization for projection printing," in Proc. SPIE Optical Microlithography XII, vol. 3679, L. Van den Hove, Ed., 1999, pp. 697-703.

[54] D. Nyyssonen, "The theory of optical edge detection and imaging of thick layers," J. Opt. Soc. Amer., vol. 72, pp. 1425-1436, 1982.

[55] D. Nyyssonen and C. P. Kirk, "Optical microscope imaging of lines patterned in thick layers with variable edge geometry," J. Opt. Soc. Amer. A, vol. 5, pp. 1270-1280, 1988.

[56] C. M. Yuan, "Efficient light scattering modeling for alignment, metrology and resist exposure in photolithography," IEEE Trans. Electron Devices, vol. 39, pp. 1588-1598, 1992.

[57] H. Tanabe, "Modeling of optical images in resists using vector potentials," Proc. SPIE, vol. 1674, pp. 637-649, 1992.

[58] X. Li, K. D. Lucas, A. L. Swecker, and A. J. Strojwas, "METROPOLE-3D: a rigorous 3D topography simulator," Proc. SPIE, vol. 3334, pp. 717-728, 1998.

[59] T. Matsuzawa, A. Moniwa, N. Hasegawa, and H. Sunami, "Twodimensional simulation of photolithography on reflective stepped substrate," IEEE Trans. Computer-Aided Design, vol. CAD-6, pp. 446-451, 1987.

[60] H. P. Urbach and D. A. Bernard, "Modeling latent-image formation in photolithography using the Helmholtz equation," J. Opt. Soc. Amer. A, vol. 6, pp. 1343-1356, 1989.

[61] P. Vincent, "Differential methods," in Electromagnetic Theory of Gratings, R. Petit, Ed. New York: Springer-Verlag, 1980.

[62] R. C. McPhedran, G. H. Derrick, and L. C. Botten, "Theory of crossed gratings," in Electromagnetic Theory of Gratings, R. Petit, Ed. New York: Springer-Verlag, 1980, sec. 7.6.

[63] M. S. Yeung, "Photolithography simulation on nonplanar substrates," Proc. SPIE, vol. 1264, pp. 309-321, 1990.

[64] G. L. Wojcik, D. K. Vaughan, and L. Galbraith, "Calculation of light scatter from structures on silicon surfaces," Proc. SPIE, vol. 774, pp. 21-31, 1987.

[65] G. L. Wojcik, D. K. Vaughan, J. Mould, F. Leon, Q. D. Qian, and M. A. Lutz, "Laser alignment modeling using rigorous numerical simulations," Proc. SPIE, vol. 1463, pp. 292-303, 1991.

[66] G. L. Wojcik, J. Mould, R. J. Monteverde, J. J. Prochazka, and J. R. Frank, "Numerical simulation of thick line width measurements by reflected light," Proc. SPIE, vol. 1464, pp. 187-203, 1991.

[67] R. Guerrieri, K. H. Tadros, J. Gamelin, and A. R. Neureuther, "Massively parallel algorithms for scattering in optical lithography," IEEE Trans. Computer-Aided Design, vol. 10, pp. 1091-1100, 1991.

[68] A. K. Wong, R. Guerrieri, and A. R. Neureuther, "Massively parallel electromagnetic simulation for photolithographic applications," IEEE Trans. Computer-Aided Design, vol. 14, pp. 1231-1240, 1995.

[69] A. K. Wong and A. R. Neureuther, "Rigorous three-dimensional time-domain finite-difference electromagnetic simulation for photolithographic applications," IEEE Trans. Semiconduct. Manufact., vol. 8, pp. 419-431, 1995.

[70] B. H. Kleemann, A. Mitreiter, and F. Wyrowski, "Integral equation method with parametrization of grating profile-theory and experiments," J. Mod. Opt., vol. 43, pp. 1323-1349, 1996.

[71] M. S. Yeung and E. Barouch, "Three-dimensional nonplanar lithography simulation using a periodic fast multipole method," Proc. SPIE, vol. 3051, pp. 509-521, 1997.

[72] M. Op de Beeck, G. Vandenberghe, P. Jaenen, F. H. Zhang, C. Delvaux, P. Richardson, I. van Puyenbroeck, K. Ronse, J. E. Lamb III, J. B. C. van der Hilst, and J. van Wingerden, "Bottom-ARC optimization methodology for $0.25 \mu \mathrm{m}$ lithography and beyond," Proc. SPIE, vol. 3334, pp. 322-336, 1998.

[73] S. Tanaka, H. Nakamura, K. Kawano, and S. Inoue, "Practical topography design for alternative phase-shifting masks," Proc. SPIE, vol. 2726, pp. 473-484, 1996. 
[74] R. A. Ferguson, A. K. Wong, T. A. Brunner, and L. W. Liebmann, "Pattern-dependent correction of mask topography effects for alternating phase-shifting masks," Proc. SPIE, vol. 2440, pp. 349-360, 1995.

[75] E. D. Palik, Ed., Handbook of Optical Constants of Solids II. New York: Academic, 1991, pp. 374-385.

[76] M. S. Yeung and E. Barouch, "Limitation of the Kirchhoff boundary conditions for aerial image simulation in 157-nm optical lithography," IEEE Electron Device Lett., vol. 21, pp. 433-435, 2000.

[77] R. B. Wu and T. Itoh, "Hybrid finite-difference time-domain modeling of curved surfaces using tetrahedral edge elements," IEEE Trans. Antennas Propagat., vol. 45, pp. 1302-1309, 1997.

[78] M. S. Yeung, "Application of the hybrid FETD-FDTD method to dispersive materials," Microwave Opt. Technol. Lett., vol. 23, pp. 238-242, 1999.

[79] T. A. Brunner and R. A. Ferguson, "Approximate models for resist processing effects," in Proc. SPIE Optical Microlithography IX, vol. 2726, G. E. Fuller, Ed., 1996, pp. 198-207.

[80] J. Li, "Model-based optical proximity correction including effects of photoresist processes," in Proc. SPIE Optical Microlithography $X$, vol. 3051, G. E. Fuller, Ed., 1997, pp. 643-651.

[81] N. Cobb, A. Zakhor, and E. Miloslavsky, "Mathematical and CAD framework for proximity correction," in Proc. SPIE Optical Microlithography IX, vol. 2726, G. E. Fuller, Ed., 1996, pp. 208-222.

[82] N. Cobb and A. Zakhor, "Experimental results on optical proximity correction with variable threshold resist model," in Proc. SPIE Optical Microlithography X, vol. 3051, G. E. Fuller, Ed., 1997, pp. 458-468.

[83] J. Zhao, J. Garofalo, J. Blatchford, E. Ehrlacher, and E. Nease, "Applications of enhanced optical proximity correction models," in Proc. SPIE Optical Microlithography XI, vol. 3334, L. Van den hove, Ed., 1998, pp. 234-244.

[84] C.-N Ahn, H.-B Kim, and K.-H Baik, "A novel approximate model for resist process," in Proc. SPIE Optical Microlithography XI, vol. 3334, L. Van den hove, Ed., 1998, pp. 752-763.

[85] E. W. Conrad, D. C. Cole, D. P. Paul, and E. Barouch, "Model considerations, calibration issues, and metrology methods for resist-bias model," in Proc. SPIE Metrology, Inspection, and Process Control for Microlithography XIII, vol. 3677, B. Singh, Ed., 1999, pp. 940-955.

[86] J. Bruce, O. Bula, E. Conrad, and A. McGuire, "Manufacturing implementation of 2D optical proximity correction," in Proc. Arch Microlithography Symp., 1999, pp. 97-112.

[87] A. Rosenbluth, D. Goodman, and B. J. Lin, "A critical examination of submicron optical lithography using simulated projection images," J. Vac. Sci. Technol. B, vol. 1, no. 4, pp. 1190-1195, 1983.

[88] D. A. Bernard, J. Li, J. C. Rey, K. Rouz, and V. Axelrad, "Efficient computational techniques for aerial imaging simulation," in Proc. SPIE Optical Microlithography IX, G. E. Fuller, Ed., 1996, vol. 2726, pp. 273-287.

[89] Y. C. Pati and T. Kailath, "Phase-shifting masks for microlithography: Automated design and mask requirements," J. Opt. Soc. Amer. A, vol. 11, no. 9, pp. 2438-2452, 1994.

[90] K. K. H. Toh, "Two-dimensional images with effects of lens aberrations in optical lithography," M.S. thesis, Univ. California, Berkeley, 1988.

[91] D. C. Cole, O. Bula, E. W. Conrad, D. S. Coops, W. C. Leipold, R. W. Mann, and J. H. Oppold, "Optimization criteria for SRAM design-lithography contribution,” in Proc. SPIE Optical Microlithography XII, vol. 3679, L. Van den hove, Ed., 1999, pp. 847-859.

[92] J. Garofalo, J. DeMarco, J. Bailey, J. Xiao, and S. Vaidya, "Reduction of ASIC gate-level line-end shortening by mask compensation," in Proc. SPIE Optical/Laser Microlithography VIII, vol. 2440, T. A. Brunner, Ed., 1995, pp. 171-183.

[93] W. Leipold, F. Baker, O. Bula, R. Leidy, and A. McGuire, "Polysilicon line end resolution enhancement: Improving yield on IBM's current and future technologies," IBM MicroNews, vol. 4, no. 3, pp. 19-23, 1998.

[94] A. Starikov, "Use of a single size square serif for variable print bias compensation in microlithography: Method, design, and practice," in Proc. SPIE Optical/Laser Microlithography II, vol. 1088, B. J. Lin, Ed., 1989, pp. 34-46.

[95] E. Barouch, U. Hollerbach, and R. R. Vallishayee, "OPTIMASK: an OPC algorithm for chrome and phase-shift mask design," in Proc. SPIE Optical/Laser Microlithography VIII, vol. 2440, T. A. Brunner, Ed., 1995, pp. 192-206.
[96] R. R. Vallishayee, S. A. Orszag, and E. Barouch, "Optimization of stepper parameters and their influence on OPC," in Proc. SPIE Optical Microlithography IX, vol. 2726, G. E. Fuller, Ed., 1996, pp. 660-669.

[97] M. Garza, E. Jackson, W. P. Shen, and N. Eib et al., "Achieving subhalf-micron I-line manufacturability through automated OPC," in Proc. SPIE Emerging Lithographic Technologies, vol. 3048, D. E. Seeger et al., Ed., 1997, pp. 382-395.

[98] B. J. Lin and P. Young, "Full-depth optical proximity correction (FD-OPC) based on E-D forest," in Proc. SPIE Optical Microlithography XII, vol. 3679, L. Van den Hove, Ed., 1999, pp. 600-606.

[99] C. M. Lim, J.-W Seo, C.-S Kang, Y.-S Park, J.-T Yoon, C.-S Lee, Chul-Seung, S.-C Moon, and B. H. Kim, "Practical methodology of optical proximity correction in subquarter-micron lithography," in Proc. SPIE Optical Microlithography XII, vol. 3679, L. Van den Hove, Ed., 1999, pp. 666-674.

[100] M. D. Levenson, N. S. Viswanathan, and R. A. Simpson, "Improving resolution in photolithography with a phase-shifting mask," IEEE Trans. Electron Devices, vol. ED-29, pp. 1828-1836, 1982.

[101] C. Spence, D. C. Cole, and B. Peck, "Using multiple focal planes to enhance depth of focus," in Proc. SPIE Optical/Laser Microlithography V, J. D. Cuthbert, Ed., 1992, vol. 1674, pp. 285-295.

[102] B. J. Lin, "The attenuated phase-shift mask," Solid State Technol., vol. 35, pp. 43-47, Jan. 1992.

[103] K. Kamon, Ph.D. dissertation, Waseda Univ., Tokyo, Japan, 1999.

[104] K. Kamon, T. Miyamoto, Y. Myoi, H. Nagata, and M. Tanaka, "Photolithography system using modified illumination," Jpn. J. Appl. Phys., vol. 32, pp. 239-243, 1993.

[105] L. W. Flanagin, V. K. Singh, and C. G. Willson, "Molecular model of phenolic polymer dissolution in photolithography," J. Polym. Sci. B, Polym. Phys., vol. 37, pp. 2103-2113, 1999.

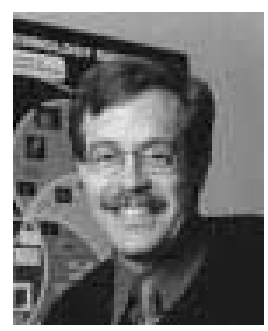

Daniel C. Cole received the Ph.D. degree in theoretical physics from the Graduate Center of the City University of New York in 1985.

He was with IBM from 1985 to 1998 on the simulation of physical processes in semiconductor technology, particularly regarding electron transport and microlithography. The last three years at IBM, he managed a department involved with semiconductor simulation development and application. In 1998, he became an associate professor in the Department of Manufacturing Engineering at Boston University. His research continues in the area of the simulation of physical processes to aid in improving the development and manufacturability of microelectronics and nanoelectronics technologies. He has numerous publications in these areas, as well as in physically fundamental work on the behavior of charged particles interacting with radiation. He also has several patents and teaches, among other things, a graduate level engineering course involving intellectual property.

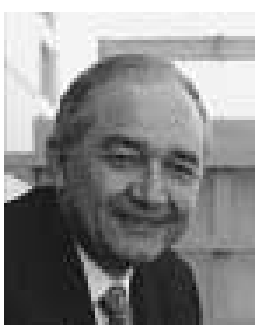

Eytan Barouch received the B.S. degree from Hebrew University, Jerusalem, in 1966 and the Ph.D. degree from State University of New York at Stony Brook in 1969, both in physics.

From 1974 to 1994, he was a Professor in the Department of Mathematics at Clarkson University. Since 1995, he has been a Professor in the Department of Manufacturing Engineering at Boston University. He has held visiting appointments at Massachusetts Institute of Technology, Kyoto University, Princeton University, and Yale University. His current research interests are in applied numerical analysis, statistical mechanics and the simulation of microelectronic devices and processes. He has published over 150 research articles. 


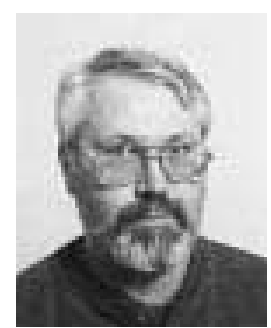

Edward W. Conrad received the B.A. degree in physics and mathematics from the University of Vermont in 1977 , followed by the M.S. degree in physics in 1982.

He joined IBM in 1978 and worked primarily on developing equipment, measurement techniques, and algorithms for optical thin film metrology until 1987. From 1987 to the present, he has been involved with optical metrology for photolithography and lithography simulation. $\mathrm{He}$ has published several articles and IBM technical disclosures, and he has several patents in the areas of metrology and photolithography. His current interests involve using detailed metrology methods to help calibrate phenomenological models used in lithography simulation programs.

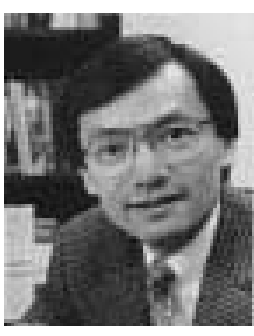

Michael Yeung received the B.S. degree in physics from California Institute of Technology, Pasadena, in 1972 and the Ph.D. degree in electrical engineering from the University of California at Berkeley in 1995.

From 1982 to 1989 , he was with Intel where he worked on optical lithography process development. From 1990 to 1995 , he was a Graduate Student Researcher in the Department of Electrical Engineering and Computer Sciences, University of California at Berkeley, where he worked on quantum optics and the fast multipole method for electromagnetic scattering. Since 1995, he has been first an Assistant Professor, and now an Associate Professor, in the Department of Manufacturing Engineering at Boston University. His current research interest is in computational electromagnetics with application to the simulation of electromagnetic scattering phenomena in microchip manufacturing. 